\title{
A Seasonal Model for West Nile Virus
}

\author{
P. Moschini ${ }^{1}$, D. Bisanzio ${ }^{2}$, A. Pugliese ${ }^{1 *}$ \\ ${ }^{1}$ Dept. of Mathematics, University of Trento, Via Sommarive 14, 38123 Povo - Trento, Italy \\ ${ }^{2}$ Big Data Institute, Nuffield Department of Medicine, University of Oxford, c/o Wellcome Trust \\ Centre for Human Genetics, Roosevelt Drive, Oxford, OX3 7BN, UK
}

\begin{abstract}
West Nile virus (WNV) is maintained in transmission cycles involving bird reservoir hosts and mosquito vectors. While several aspects of the infection cycle have been explored through mathematical models, relatively little attention has been paid to the theoretical effect of seasonal changes in host and vector densities. Here we consider a model for the transmission dynamics of WNV in a temperate climate, where mosquitoes are not active during winters, so that infection dynamics can be described through a sequence of discrete growing seasons. Within-season host and vector demography is described through phenomenological functions of time describing fertility, mortality and migration. Over-wintering of infection is assumed to occur through diapausing mosquito females, with or without vertical transmission.

We introduce a parameter $S_{0}$ that, similarly to $R_{0}$ but easier to compute, yields a threshold condition for infection persistence in this semi-discrete setting. Then we study the possible dynamical behavior of the model, by exploring parameter values through a Latin Hypercube Sampling and accepting only those values yielding solutions respecting a few conditions obtained from the qualitative patterns observed in yearly patterns of mosquito abundance and virus prevalence.

For some parameters the posterior distribution is rather narrow, implying that simple qualitative agreement with data can yield information on parameter difficult to estimate directly. For other parameters, the posterior distribution is instead similar to the prior. Simulations of multi-year dynamics after a first introduction of the virus always asymptotically result, if $S_{0}>1$, in a pattern of yearly identical infections; however, their amplitude may be very different, even for the same value of $S_{0}$, in correspondence to the uncertainties about several parameters.
\end{abstract}

Keywords and phrases: seasonal epidemic model, West-Nile virus, overwintering

Mathematics Subject Classification: 92D30

\section{Introduction}

West Nile Virus (WNV) is a mosquito-borne virus of the Flaviviridae family, which is a neuropathogen for humans, horses and birds. West Nile virus is maintained by enzootic cycle involving mosquitoes, vectors, and reservoir birds. Humans, horses and other mammals are considered as dead-end host for the virus $[11,30]$.

Because of their local abundance, vector competence in the laboratory [68] and frequent reports of

${ }^{*}$ Corresponding author. E-mail: andrea.pugliese@unitn.it 
infection with WNV in nature [3,4], several mosquito species have been tested for WNV (see Center for Disease Control $[\mathrm{CDC}]$ ) and have thus been considered as vectors. Vector species which bites mammals can act as bridge vectors causing spillover in human population. Nevertheless. Culex (Cx.) pipiens species is considered, on the basis of the data documenting feeding on birds and mammals [29], as the main epizootic and endemic vector of WNV in Europe [31] and Northeastern and North Central United States $[4,52]$. This is one of the most widespread mosquito species, with a distribution covering all temperate regions.

In competent birds viremia lasts 1-7 days post infection (depending on infected species). During this period, birds are able to transmit WNV to susceptible mosquitoes and, subsequently, develop life-long immunity [48]. Birds can be classified as highly competent hosts (HCH) or mildly competent hosts $(\mathrm{MCH})[13,36]$, according to the duration of viremia.

In USA the first reported human outbreak of West Nile Disease (WND) was reported in 1999 in New York City. Then the virus spread into many US states and in few years became endemic.Since the first reported cases, human incidence of WND has shown oscillation. In 2012 there was peak in incidence [15] after three years of low circulation in humans.

In Southern Europe the dynamics of the infection seems to be different. Outbreaks were reported in several areas of Europe, followed by spatial expansions in the following years and decreases or even disappearances in the initial areas. The infection prevalence seems to move like a wave during the years $[25]$

The model we have developed is not tailored to a specific area, but assumes a generic temperate climate; its aim is to investigate whether different parameter values can lead to different qualitative behaviors, reminiscent of the different dynamics of WNV infection observed in different areas.

There exists an extensive literature of mathematical models for WNV.

As far as we know, the first models were presented in [67], where a difference equation model for West Nile virus was proposed, while systems of ordinary differential equation modeling WNV transmission in the mosquito and bird populations were proposed in $[12,19,72]$, on the basis of the classical Ross-McDonald malaria model $[2,44]$. Their study has been extended in several directions, such as the spatial diffusion of the infection [41,42,46], more detailed mathematical analysis [32], heterogeneity in the competence of reservoir species $[1,21,66]$, also involving human and equine population in the model [39].

Only few papers [20] take into account the seasonality of the species involved in the transmission, although it is well known that climate and weather strongly influence the biology of vectors in different forms like changes in reproduction, population size, and blood feeding [62].

In some models $[5,6]$ seasonality has been introduced by assuming sinusoidal fluctuations in transmission coefficients or other parameters, with the main aim of arriving at a consistent definition of $R_{0}$ in a periodic environment. Here we instead aim at building a reasonably realistic, but still rather simple system, capable of yielding qualitative conclusions about patterns in infection dynamics. In this respect one fundamental feature of mosquito-borne infections in temperate climates is that in winter there are no active mosquitoes, thus no infection transmission. Correspondingly, the model will be based on a system of differential equations describing demography and infection transmission during the summers coupled by rules for population survival and stage transition during winters.

Indeed, the mechanisms that allow for the efficient overwintering and subsequent amplification of West Nile virus have not been elucidated. In the literature, different explanations have been proposed for the overwintering of WNV: it may occur through infection amplification during bird migrations, but this is not completely understood [22,56,71]. Otherwise, overwintering could be due to mosquitoes: as they generally survive winter as diapausing adults, it is possible that mosquito larvae, infected vertically, would then enter diapause as they develop into adults, without taking a blood meal, and survive winter; they could then transmit the infection in the following season $[8,28]$. Alternatively, adult mosquitoes infected by feeding on a infected avian host could then enter diapause and survive the winter [53]. Indeed, Bailey 
et al. [7] analyzed data providing evidence to support the theory that a significant number of diapausing Culex pipiens, which have taken a prehibernation blood meal, do not develop eggs and can survive the winter at rate comparable to diapausing non blood fed mosquitoes.

In this paper, we will assume that WNV overwintering occurs through the mosquito population, according to either one of the above mechanisms.

The main aim of this paper is to study the dynamical behavior of the resulting models from the qualitative and semi-quantitative point of view. First, we elucidate the conditions under which the infection can persist, persistence depending on the threshold quantity $S_{0}$. Second, we simulate the models that include the different over-wintering mechanisms using parameter values sampled from a feasible set and select those values that yield outcomes compatible with known qualitative features of mosquito population and WNV infection. This allows us to examine the annual and multi-annual dynamical behaviors that are expected, depending on the overwintering mechanism, as a consequence of the seasonality of mosquito demographic traits.

We do not aim at fitting a specific dataset, but rather at exploring the effect of generic seasonal patterns on the dynamic of WNV infection. Because of this focus, the model is greatly simplified in other respects, most importantly we will consider only one type of reservoir hosts (birds) without distinguishing between hosts differing in competence. This issue has been examined in detail in several recent models [50,66].

\section{Modeling the population dynamics}

We start by modeling the populations involved in the transmission, birds and mosquitoes, in a disease free state. We divide the years in two periods: one (named $(0, T)$ ) during which infection transmission occurs due to mosquitoes being active. A second period $(T, 365)$ (having chosen 1 day as the time unit) where no infections occur.

\subsection{Bird population}

The bird population is modeled taking into account, in an extremely simple way, the breeding season and the outgoing migration. Let $b_{1}$ the fertility, $d_{1}$ the mortality and $m_{1}$ the outgoing migration rate of the bird population. We assume that death rate is constant over the summers, while births and migration are concentrated in part of the season. Precisely, we assume that at time $t=0$ the migrating birds have already arrived at the region being modeled and the breeding season is just starting. Egg hatching occurs, at constant rate, up to time $t_{1}^{*}$; after $t_{1}^{*}$, as there are no births or immigrations, the bird population decreases because of deaths, and, beyond time $t_{2}^{*}$, also because of outgoing migration.

The bird population dynamics is then described by the following equation:

$$
\dot{N}_{B}(t)=N_{B}(t)\left(P(t) b_{1}-d_{1}-Q(t) m_{1}\right) \quad N_{B}(0)=k_{B}
$$

where

$$
P(t)=\left\{\begin{array}{ll}
1 & \text { if } t<t_{1}^{*} \\
0 & \text { if } t>t_{1}^{*}
\end{array} \quad \text { and } \quad Q(t)= \begin{cases}1 & \text { if } t<t_{2}^{*} \\
0 & \text { if } t>t_{2}^{*}\end{cases}\right.
$$

with $0<t_{1}^{*}<t_{2}^{*}<T$.

In the left panel of Figure 2 we show the solution of equation (2.1) with the parameter values and initial condition $N_{B}(0)=k_{B}$ that will be used in the rest of the paper.

\subsection{Mosquito population}

Mosquitoes go through four separated and distinct stages of its life cycle: egg, larva, pupa and adult. Only female mosquitoes bite animals, requiring a blood meal to obtain the necessary nutrients for the development and maturation of eggs.

In what follows, we consider only females and neglect explicit consideration of immature stages; instead, because of its importance for infection transmission, we take into account the gonotrophic cycle by 
dividing adult females into two stages: the compartment of resting mosquitoes, $G(t)$, composed by the mosquitoes that, after a blood meal, need a period to digest and metabolize it; $F(t)$, the adults searching for hosts on which to feed on. Their dynamics can be described by the following equations

$$
\left\{\begin{array}{l}
\dot{F}(t)=f(t) G(t)-d(t) F(t)-\alpha F(t)+\epsilon G(t) \\
\dot{G}(t)=\alpha F(t)-\epsilon G(t)-d(t) G(t)
\end{array}\right.
$$

In the model, $\alpha$ is the rate at which mosquitoes leave the searching stage (as they have completed a blood meal); hence $1 / \alpha$ is the mean length of the questing period: it is assumed that its length does not depend on host density, as their number is never a limiting resource. Similarly the mean length of the resting period is $\frac{1}{\epsilon}$, as the exit rate from the compartment $G(t)$ is $\epsilon$.

The rate $f(t)$ at which new adults enter the population is $f(t)=b(t)(1-p(t))$ where $b(t)$ represents the fertility and $p(t)$ the probability that new adults enter diapause at time $t$; finally, $d(t)$ the mortality.

These functions are assumed to be periodic, in order to reproduce the seasonal dynamics of mosquito population. We used

$$
\begin{aligned}
& b(t)=b_{2}\left(0.75+0.25 \sin \left(\left(t+s_{1}\right) \frac{\pi}{L_{1}}\right)\right) \\
& p(t)=0.5+\frac{1}{\pi} \arctan \left(A\left(t-s_{2}\right)\right) \\
& d(t)=d_{2}\left(1+B \sin \left(\left(t+s_{3}\right) \frac{\pi}{L_{3}}\right)\right)
\end{aligned}
$$

These functions have been obtained by coupling function modeling mosquito demography responses to temperature and photo-period [64] to an average temperature cycle in a warm-temperate climate. In Figure 1 we plot the functions with the parameter values that have been used in the rest of the paper.

Note that in model (2.3) it is assumed that new adult mosquitoes develop immediately from newly laid eggs. Of course, this is not biologically realistic, as some time is necessary to go through all the immature stages (eggs, larvae, pupae); moreover, the developmental time will also depend on temperature [45] and thus will vary during the season. For the sake of simplicity, we neglect this important factor in the present analysis, but we simply sketch in Appendix B how it could be handled.

In the right panel of Figure 2 we show the result of simulating the model using the functions and parameter values described above. It seems that the qualitative features of mosquito dynamics resemble the data on densities of Culex pipiens mosquitoes obtained for United States [29] and for Italy [64].

\section{The model of infection dynamics}

We build a model using a standard SIR epidemiological framework to model enzootic transmission between an avian population and the Culex Pipiens mosquito population. The avian hosts are divided into classes of susceptible $\left(S_{B}\right)$, infected $\left(I_{B}\right)$ and recovered $\left(R_{B}\right)$ individuals, so the total population size is $N_{B}(t)=$ $S_{B}(t)+I_{B}(t)+R_{B}(t)$. Newborn birds are all susceptibles (Hamer et al, 2008); after becoming infected, birds recover at rate $\gamma$ and develop life-long immunity to further West Nile infection. They can also die because of West Nile infection at rate $\mu_{W N}$.

It is assumed that mosquitoes do not recover from infection with WNV. The population is divided into four compartments, i.e. $S_{M}, E_{M}, G_{M}$ and $I_{M}$. $S_{M}$ and $I_{M}$ represent the questing mosquitoes that are, respectively, susceptible and infected from West Nile virus. $E_{M}$ and $G_{M}$ represent those in the gonotrophic cycle that have or have not been infected.

When a susceptible mosquito bites (at rate $\alpha \frac{I_{B}}{N_{B}}$ ) an infected bird, it becomes infected with probability $\beta_{M}$ and enters the gonotrophic cycle. We assume that the latent period is completed during the resting period, so it will be infectious by the time of the following feeding period.

Both $E_{M}$ and $G_{M}$ produce eggs. $E_{M}$ mosquitoes give birth to already infected mosquitoes with probability $\nu$ of vertical transmission. 

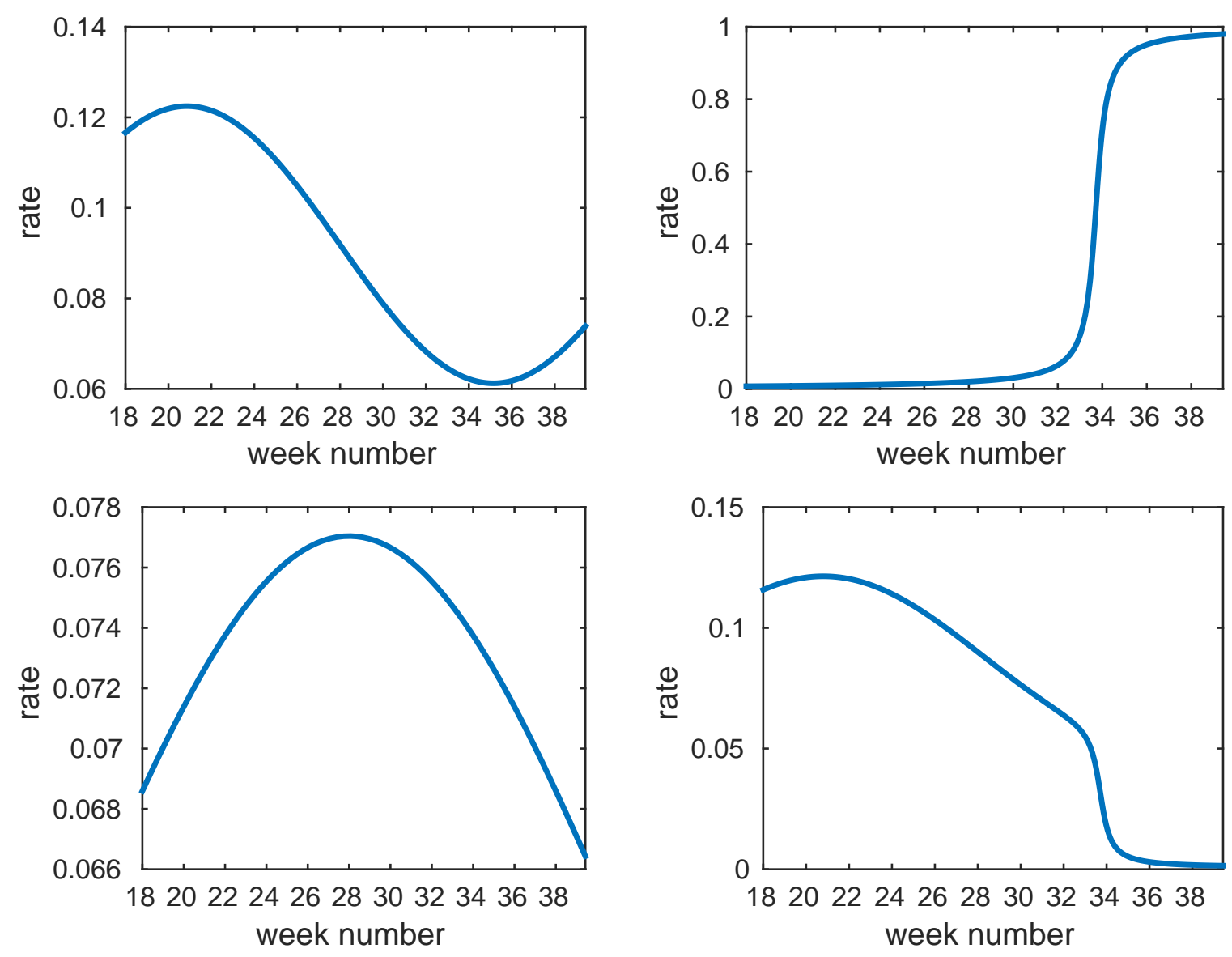

FIGURE 1. fertility rate function $b(t)$, diapause rate function $p(t)$, mortality rate function $d(t)$ and recruitment rate function $f(t)$ using (2.4) with parameter values $A=0.4, B=$ $0.2, s_{1}=30, s_{2}=110, s_{3}=20, L_{1}=100$ and $L_{3}=180$
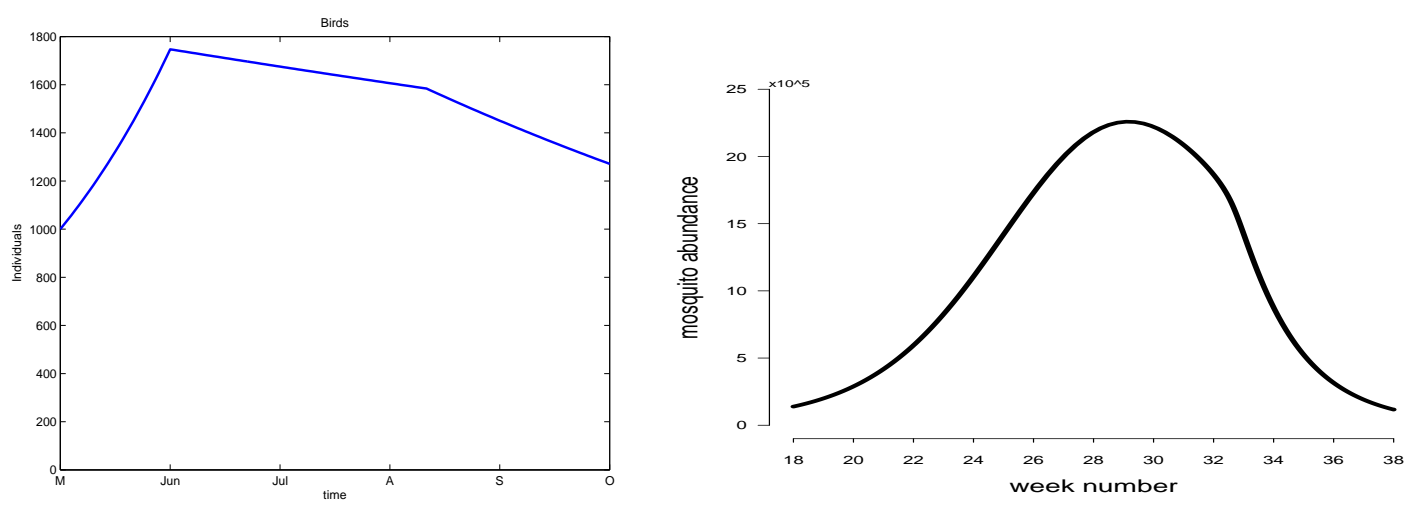

Figure 2. Left panel: a simulation of model $(2.1)$ with $d_{1}=1.37 \cdot 10^{-3}, b_{1}=0.023$, $m_{1}=0.03, t_{1}^{*}=30, t_{2}^{*}=120$, all values in days or $(\text { days })^{-1}$. Right panel: a simulation of model (2.3)-(2.4) with parameter values as in Fig. 1. 
When a bird is bitten by an infected mosquito (compartment $I_{M}$ ), it will become infected with a probability $\beta_{B}$. The relative flow chart is shown in Figure 3. The following system of differential equation,

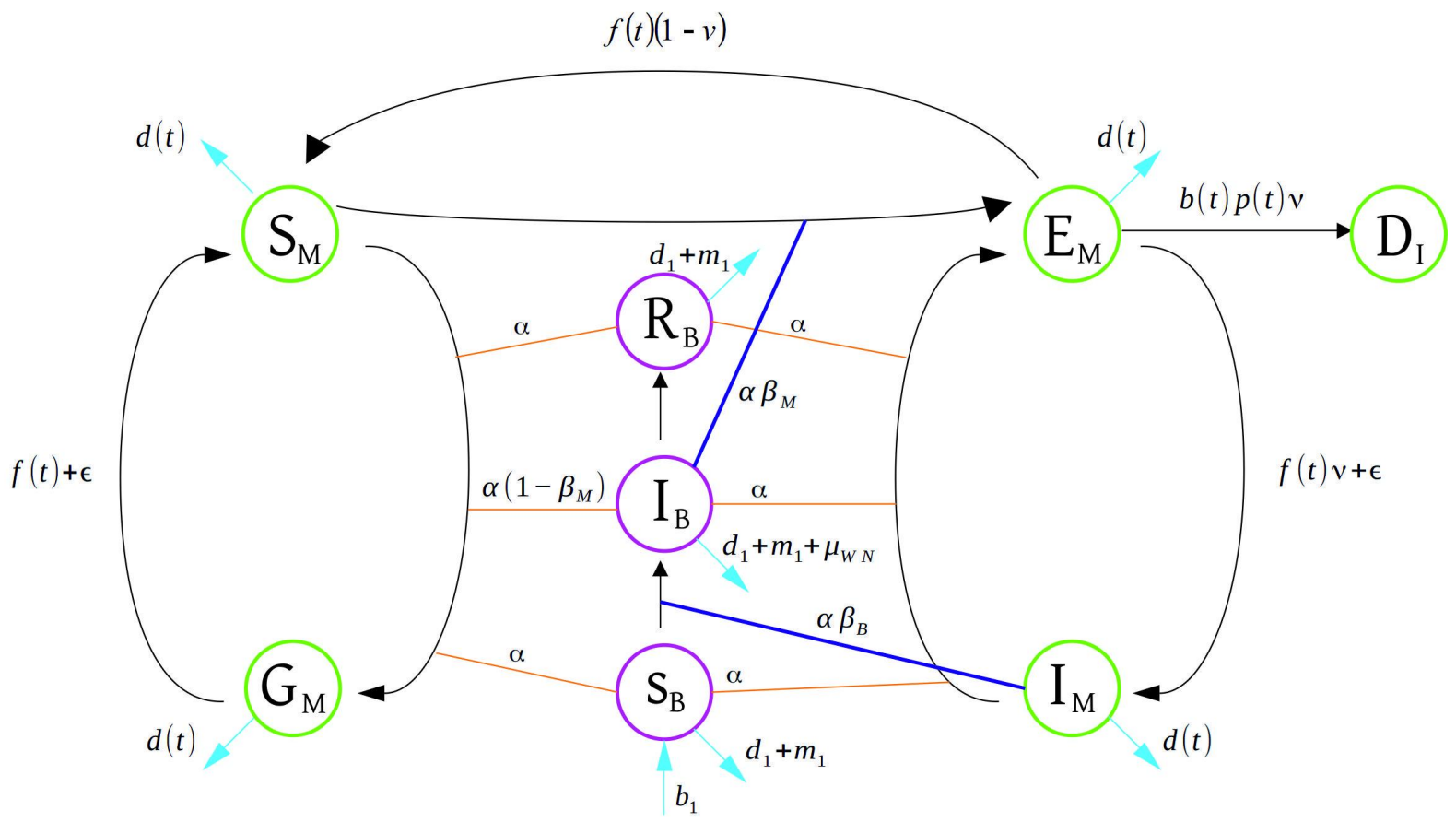

FiguRE 3. Flow chart of the model.

with $t \in[0, T]$, describes West Nile virus transmission between vectors and hosts, and incorporate the vector biting rate $(\alpha)$, the transmission rates $\beta_{B}$ from vector to host and $\beta_{M}$ from host to vector during the summer season of length $T$.

$$
\left\{\begin{array}{l}
\dot{S}_{B}(t)=P(t) b_{1} N_{B}(t)-d_{1} S_{B}(t)-\alpha \frac{\beta_{B} I_{M}(t)}{N_{B}(t)} S_{B}(t)-Q(t) m_{1} S_{B}(t) \\
\dot{I}_{B}(t)=\alpha \frac{\beta_{B} I_{M}(t)}{N_{B}(t)} S_{B}(t)-\gamma I_{B}(t)-\mu_{W N} I_{B}(t)-d_{1} I_{B}(t)-Q(t) m_{1} I_{B}(t) \\
\dot{R}_{B}(t)=\gamma I_{B}(t)-d_{1} R_{B}(t)-Q(t) m_{1} R_{B}(t) \\
\dot{S}_{M}(t)=f(t)\left((1-\nu) E_{M}(t)+G_{M}(t)\right)-d(t) S_{M}(t)-\alpha S_{M}(t)+\epsilon G_{M}(t) \\
\dot{E}_{M}(t)=\alpha \frac{\beta_{M} I_{B}(t)}{N_{B}(t)} S_{M}(t)-\epsilon E_{M}(t)-d(t) E_{M}(t)+\alpha I_{M} \\
\dot{G}_{M}(t)=\alpha \frac{S_{B}(t)+R_{B}(t)+\left(1-\beta_{M}\right) I_{B}(t)}{N_{B}(t)} S_{M}(t)-\epsilon G_{M}-d(t) G_{M}(t) \\
\dot{I}_{M}(t)=f(t) \nu E_{M}(t)+\epsilon E_{M}(t)-d(t) I_{M}(t)-\alpha I_{M}(t) \\
\dot{D}_{I}(t)=b(t) p(t) \nu E_{M}(t)
\end{array}\right.
$$

In (3.1), $P(t)$ and $Q(t)$ are the step functions described in (2.2). Furthermore, the compartment $D_{I}$ represents the number of vertically infected mosquitoes that enter diapause. We do not instead take into account the number of not infected diapausing females, as these are irrelevant for the dynamics of infection. All parameters are described in Table 1. 


\begin{tabular}{|c|c|c|c|}
\hline Parameter & range/value & description & references \\
\hline$b_{1}$ & 0.023 & birth rate of birds & elaboration from [55] \\
\hline$d_{1}$ & 0.0014 & death rate of birds & elaboration from [55] \\
\hline$m_{1}$ & 0.003 & out-going migration rate of birds & guess \\
\hline$k_{B}$ & $10^{3}$ & bird density at the start of summer & elaboration from [55] \\
\hline$b_{2}$ & $(0,0.5]$ & coefficient of fertility function for mosquitoes & {$[18,45,49]$} \\
\hline$d_{2}$ & $(0,0.2]$ & coefficient of mortality function for mosquitoes & {$[18,45,49]$} \\
\hline$k_{M}$ & {$\left[10^{4}, 2 \cdot 10^{5}\right]$} & mosquito density at the start of the summer & {$[17,49]$} \\
\hline$\alpha$ & {$[0.2,0.7]$} & biting rate of mosquitoes & {$[61,72]$} \\
\hline$\beta_{B}$ & {$[0,1]$} & vector to host transmission probability & whole feasible interval \\
\hline$\beta_{M}$ & {$[0,1]$} & host to vector transmission probability & whole feasible interval \\
\hline$\gamma$ & {$[0.1,0.3]$} & recovery rate of birds & {$[37]$} \\
\hline$\mu_{W N}$ & {$[0,0.5]$} & death rate of birds due to $W N V$ infection & {$[37]$} \\
\hline$\epsilon$ & {$[0,0.2]$} & rate at which mosquitoes end the egg-laying stage & {$[27]$} \\
\hline$\nu$ & {$[0,0.1]$} & vertical transmission probability & {$[54]$} \\
\hline$t_{1}^{*}$ & 30 & end of breeding period & guess \\
\hline$t_{2}^{*}$ & 120 & start of outgoing migration & guess \\
\hline
\end{tabular}

TABLE 1. Parameter values and meaning. The rates have units per day, while densities are per hectare.

This system of equation will hold for each summer season $n=1,2, \ldots$ Its initial conditions depend on the final conditions of the system of the previous summer, as explained in the next Section.

\section{Overwintering and disease persistence in the multi-year model}

We denote by $S_{B}^{n}(t), I_{B}^{n}(t) \ldots, t \in[0, T], n=1,2, \ldots$ the densities of birds or mosquitoes in the different compartments depicted in Fig. 3 at time $t$ of summer $n$. These variables will satisfy equations (3.1) for each $n$ and $t \in[0, T]$. The initial conditions will depend on the overwintering mechanism of West Nile virus.

As discussed in the Introduction, we consider two different mechanisms for overwintering, assuming either survival of unfed diapausing adults, infected through vertical transmission; or survival of adult mosquitoes that have been infected by feeding on an infected bird. In both cases, we assume that all infected bird recover during the winter and, if alive, they will be immune at the beginning of the following year; $\rho$ is the birds' probability of surviving winter.

We assume that the total density of birds $\left(k_{B}\right)$ and of mosquitoes $\left(k_{M}\right)$ are the same at the beginning of each summer. Furthermore, no mosquito will be in the gonotrophic cycle or in diapause: hence $S_{M}^{n}(0)+$ $I_{M}^{n}(0)=k_{M}$.

In summary, the initial conditions are

$$
\left\{\begin{array}{l}
S_{B}^{n}(0)=k_{B}-R_{B}^{n}(0) \\
I_{B}^{n}(0)=0 \\
R_{B}^{n}(0)=\rho\left(I_{B}^{n-1}(T)+R_{B}^{n-1}(T)\right) \\
S_{M}^{n}(0)=k_{M}-I_{M}^{n}(0) \\
E_{M}^{n}(0)=0 \\
G_{M}^{n}(0)=0 \\
D_{I}^{n}(0)=0
\end{array}\right.
$$

We still have to assign $I_{M}^{n}(0)$; this will depend on the overwintering mechanism. In the first case (transmission through unfed diapausing adult females) we obtain

$$
I_{M}^{n}(0)=\delta_{v} D_{I}^{n-1}(T)
$$


where $\delta_{v}$ is the probability of surviving winter for a diapausing female. Note that $D_{I}^{n-1}(T)>0$ only if the probability of vertical transmission $\nu$ is positive. This will be then assumed; otherwise, infection persistence would be impossible.

The second overwintering is that normal adults can survive winter, yielding

$$
I_{M}^{n}(0)=\delta_{h} I_{M}^{n-1}(T)
$$

where now $\delta_{h}$ is the probability of surviving winter for adult females.

In this case we assume, for the sake of simplicity, that $\nu=0$, as vertical transmission is not needed for infection persistence.

\section{1. $S_{0}$ : a long-term threshold quantity}

The solution of (3.1) with initial conditions (4.1) can be seen as a function $y\left(t ; I_{M}(0), R_{B}(0)\right)$ where $y(t)$ is the vector

$$
\left(S_{B}(t), I_{B}(t), R_{B}(t), S_{M}(t), E_{M}(t), G_{M}(t), I_{M}(t), D_{I}(t)\right)
$$

as the initial conditions (4.1) are fixed but for the values $I_{M}(0)$ and $R_{B}(0)$.

One can then summarize the whole system as a discrete map

$$
\left(I_{M}^{n}(0), R_{B}^{n}(0)\right)=F\left(I_{M}^{n-1}(0), R_{B}^{n-1}(0)\right) .
$$

If (4.2) hold, the map is

$$
F(Q, R)=\left(\delta_{v} y_{8}(T ; Q, R), \rho\left(y_{2}(T ; Q, R)+y_{3}(T ; Q, R)\right)\right) .
$$

Using instead (4.3), we have

$$
F(Q, R)=\left(\delta_{h} y_{7}(T ; Q, R), \rho\left(y_{2}(T ; Q, R)+y_{3}(T ; Q, R)\right)\right) .
$$

It is then easy to see that the persistence of the disease is determined by the value of $\rho\left(F^{\prime}(0,0)\right)$, the spectral radius of the Jacobian matrix of $F ; F$ can be seen as the the Poincaré map of the periodic (because of the sequence of summers and winters) system. The infection will persist over the years when $\rho\left(F^{\prime}(0,0)\right)>1$, while it will go extinct for $\rho\left(F^{\prime}(0,0)\right)<1[6,70]$.

As shown in Appendix A, $\rho\left(F^{\prime}(0,0)\right)>1$ if and only if the same is true for its first entry, $F^{\prime}(0,0)_{11}$; thus it is convenient to define

$$
S_{0}=F_{11}^{\prime}(0,0)=\frac{\partial I_{M}^{n}(0)}{\partial I_{M}^{n-1}(0)} .
$$

The infection will persist [go extinct] when $S_{0}>[<] 1$.

In Appendix A, it is also shown how $S_{0}$ can be computed by differentiating (3.1) with respect to the initial condition $Q=I_{M}(0)$ to obtain the variational system

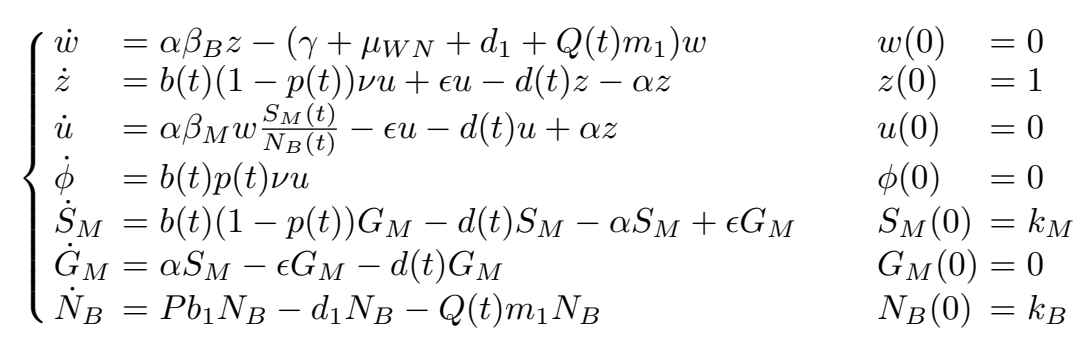

Here $w=\frac{\partial I_{B}}{\partial Q}, z=\frac{\partial I_{M}}{\partial Q}, u=\frac{\partial E_{M}}{\partial Q}, \phi=\frac{\partial D_{I}}{\partial Q}=$. The derivatives of the other variables are not needed to compute $S_{0}$. 
Finally $S_{0}$ can be obtained from (4.7) in the two cases, either as

$$
S_{0}^{v}=\delta_{v} \phi(T)
$$

or as

$$
S_{0}^{h}=\delta_{h} z(T)
$$

\section{Model parameters}

\subsection{Method of selecting acceptable values}

The resulting model is rather rich of parameters. There exist several information on the demography of Culex spp. mosquitoes in the laboratory under different environmental conditions [18, 23, 27, 43, 58], and of several passerine species [55], on WNV competence of several bird species $[37,57,68]$ and of mosquitoes [61].

While detailed information are essential for parameter estimates in a model for a specific context, our approach is to build a model that describes a generic scenario for WNV infection in a warm-temperate climate, but in which species composition (hence WNV competence) may differ, as well as yearly environmental conditions. Hence, after setting a few parameters (bird demography, season length) to reasonable values (but they can clearly be scaled), we sampled all other parameter values looking for those yielding solutions compatible with a few accepted properties, in order to encompass different plausible scenarios of WNV dynamics.

Precisely, we set the parameters of bird demography to average values of passerine species [55] with an average life of an adult bird 2 years, implying that the death rate is $d_{1}=1 /(2 \cdot 365$ days $)$.

The summer is considered to last 150 days from May 1 to September 30; thus, survival over the summer is approximately 0.81 ; consistently, $\rho$ (survival over winter) has to be set to 0.74 .

We assume that the breeding season starts on May 1 and ends after 30 days, during which period every couple of adult birds produces two offsprings; this means that the birth rate is $b_{1}=0.023(\text { days })^{-1}$. We assume that migration starts at the end of August with a rate differing from species to species and from region to region. As a value that produces realistic population values, we selected $m_{1}=0.03(\text { days })^{-1}$.

As for the other parameters, we used literature data to obtain ranges for most of them (see Table 1). Concerning the probabilities of transmission from infected mosquito to susceptible birds, or from infected bird to susceptible mosquito, it is well known that they depend in a fundamental way on the bird species involved $[37,57,61,68]$; Malik et al. [47] used this information together with an estimate of species composition of the birds being biten by mosquitoes to produce aggregate estimates of transmission probability. Infection transmission will also depend on host feeding patterns $[63,66]$; as we wish to explore all species compositions compatible with observed patterns of WNV infection, we instead let these parameters vary in the whole feasible range $(0,1)$. Finally, we use the Latin Hypercube sampling [51] (with uniform priors on the prescribed range for each variable) to obtain samples of acceptable parameter values. A sample was deemed to be acceptable, if it gave rise to solutions satisfying the constraints specified below. Note that Malik et al. [47] used Latin Hypercube sampling to perform a sensitivity analysis of $R_{0}$ on parameter values; while the sampling procedure is similar (using however normal and gamma distributions for parameters), their objective is very different from ours.

The sample was realized in two stages. First of all, we generated 10,000 samples of the parameters involving mosquito population, $b_{2}, d_{2}$ and $k_{M}$, obtaining a $(10,000 \times 3)$ matrix. For each sample, we solved (2.1) and (2.3) and selected only those samples such that the solution satisfied the following two constraints related to population dynamics without infection:

a) peak density of mosquito population has to be around (this is made precise below) 1,000 times the peak density of birds. The value 1,000 has been calculated using the mean number of mosquitoes 
collected at peak level and bird density obtained from field work data published in [40]. The precise constraint is that a simulation acceptable only if

$$
666 \leq \frac{\max _{t \in(0, T)}\left(N_{M}(t)\right)}{\max _{t \in(0, T)}\left(N_{B}(t)\right)} \leq 1,500
$$

b) Mosquito density at the start (early May) and the end (late September) of the season has to be about $5 \%$ of the peak density [64]. Precisely, this has been implemented as the following constraint on simulations:

$$
0.035 \leq \frac{N_{M}(0)}{\max _{t \in(0, T)}\left(N_{M}(t)\right)}, \quad \frac{N_{M}(T)}{\max _{t \in(0, T)}\left(N_{M}(t)\right)} \leq 0.07 .
$$

Then each selected parameter set of $b_{2}, d_{2}$ and $k_{M}$ (a subset of the original 10,000) was combined with another matrix of samples of dimensions $(3,000 \times 7)$ including the parameters involved in the transmission $\alpha, \beta_{B}, \beta_{M}, \gamma, \mu_{W N}, \epsilon$ and $\nu$.

For each resulting combination of parameters, we solved (3.1) with initial conditions typical of the situation after some years. Thus, a certain proportion of the birds will be immune (because of infections having occurred in the previous years) and a small fraction of the emerging mosquitoes will be infected, after over-wintering:

$$
\begin{aligned}
S_{B}^{1}(0) & =0.6 k_{B} \\
I_{B}^{1}(0) & =0 \\
R_{B}^{1}(0) & =0.4 k_{B} \\
S_{M}^{1}(0) & =0.999 k_{M} \\
G_{M}^{1}(0) & =0 \\
E_{M}^{1}(0) & =0 \\
I_{M}^{1}(0) & =0.001 k_{M}
\end{aligned}
$$

The constraints required of the solutions were:

1. the peak of infected mosquitoes is a couple of weeks after the peak of the total population of the mosquitoes (middle of July because of the assumptions on mosquito population dynamics);

2. enough susceptible birds are left at the end of season;

3. if no vertical transmission is assumed (see Section (4), infected mosquitoes at the end of the season must be more than at the start (otherwise the infection could not ever persist).

Precisely, if $t^{*} \in[0, T]$ is the peak time, i.e.

$$
I_{M}\left(t^{*}\right)=\max _{t \in(0, T)}\left(I_{M}(t)\right)
$$

then the constraints are

1.

$$
t^{*}>\frac{3}{5} T
$$

2.

$$
S_{B}(T)>0.02 k_{B}
$$

3.

$$
I_{M}(T)>I_{M}(0) \quad \text { (when no vertical transmission is assumed). }
$$

This rejecting procedure left us with 198 sets of parameters that satisfy the constraint for the model without vertical transmission, and 3,271 for the model with vertical transmission. 

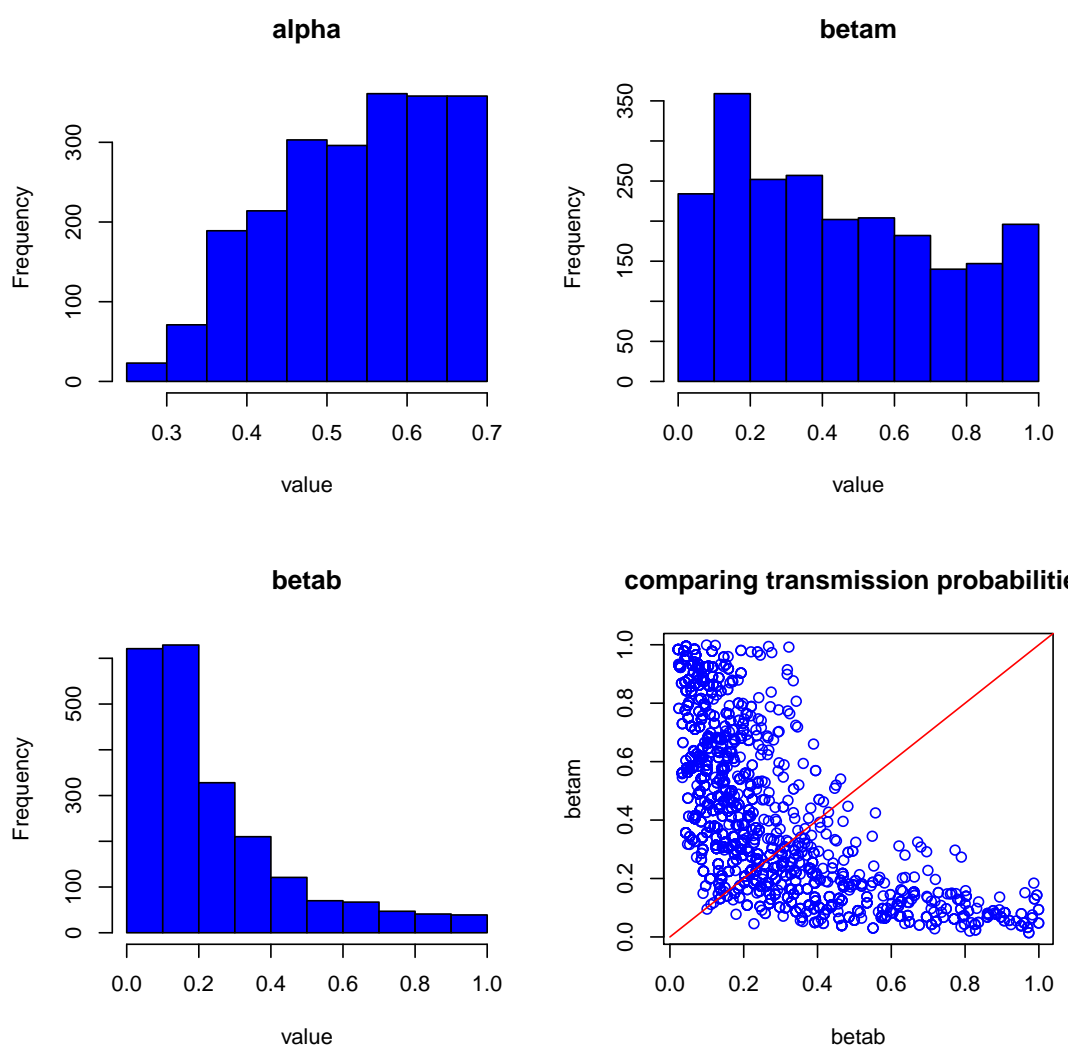

comparing transmission probabilities

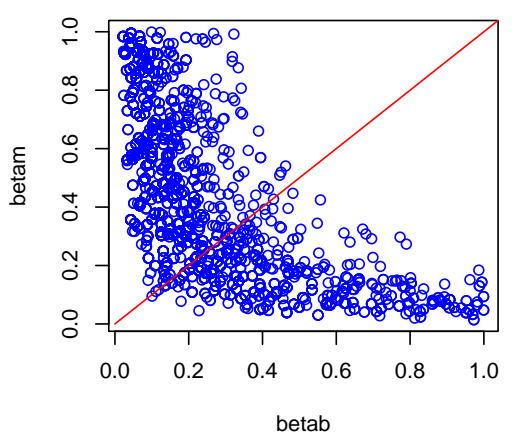

FiguRE 4. Posterior distribution of some model parameters for the case with vertical transmission

\subsection{A posteriori parameter distributions}

The procedure produced a posteriori distributions of all parameters. While for several parameters the posterior distribution is quite similar to the uniform prior, for others $\left(\beta_{B}, \beta_{M}\right.$ and $\alpha$ in Figure 4$)$ one sees an apparent mode with more likely values for the biting rate $\alpha$ in the interval $[0.45,0.7]$, and for the transmission probabilities $\beta_{B}$ in $(0,0.2]$ and $\beta_{M}$ in $[0.1,0.2]$. The fourth panel in the same Figure shows a 2-dimensional plot of the joint distribution of $\beta_{B}$ and $\beta_{M}$. An expected, strong negative correlation emerges between the estimates of the two parameters with a higher frequency of samples with $\beta_{M}>\beta_{B}$ (probability of transmission bird-to-mosquito higher than vice versa), in agreement with values used in most models.

The results for the case without vertical transmission are similar. We compare in Fig. 5 the box-plots of the estimates of the transmission probabilities in the case with or without vertical transmission. 


\section{Transmission probabilities}
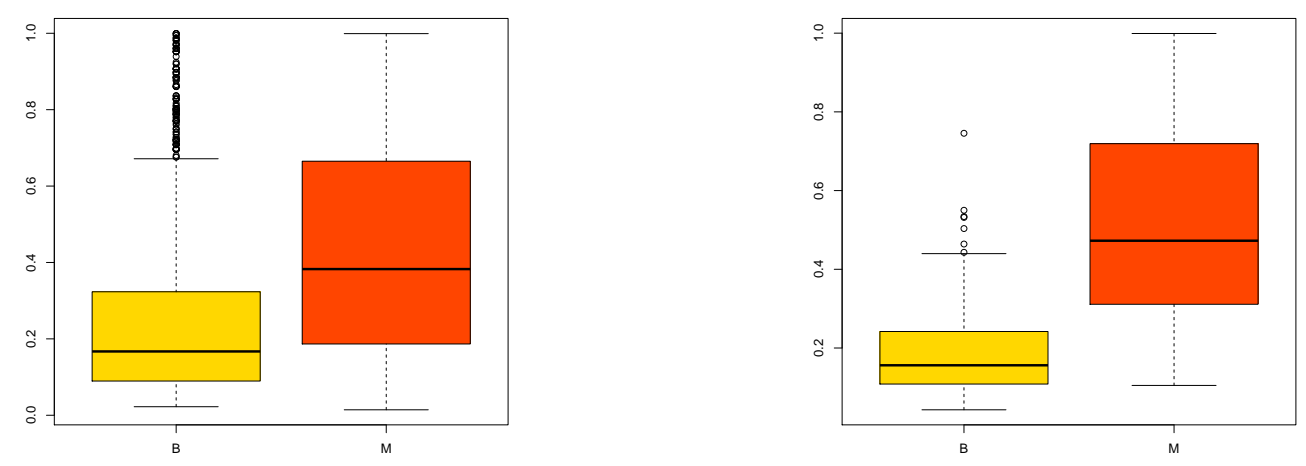

FiguRE 5 . in yellow the values of $\beta_{B}$ are shown and in orange the values of $\beta_{M}$ of the model with/without vertical transmission (left/right respectively)

\section{Multi-year simulations}

We simulated the model in the case of an initial introduction of the infection starting from one migrating bird that had been infected in its winter grounds. Thus the initial conditions are

$$
\left\{\begin{array}{l}
S_{B}^{1}(0)=k_{B}-1 \\
I_{B}^{1}(0)=1 \\
R_{B}^{1}(0)=0 \\
S_{M}^{1}(0)=k_{M} \\
G_{M}^{1}(0)=0 \\
E_{M}^{1}(0)=0 \\
I_{M}^{1}(0)=0
\end{array}\right.
$$

With these initial conditions, we ran several simulations of the model, each time choosing the parameter values from the posterior distribution shown in the previous Section; the parameter $\delta_{v}$ (or $\delta$, according to the over-wintering scheme considered) has been adjusted so as to yield the value of $S_{0}$ that had been prescribed for a set of simulations.

In Figure 6 we give an illustration of the difference in solutions that share the same value of $S_{0}$ but are obtained with different parameter values, all satisfying the constraints (5.1)-(5.5) concerning annual patterns. Precisely, Figure 6 displays 20 years of simulations obtained with the first six parameter combinations selected (for the sake of clarity, they are listed in Table 2), all with $S_{0}=2$. These simulations include vertical transmission, while the case without vertical transmission is illustrated in Appendix C.

One may note that, although they all reach persistence (as expected since $S_{0}>1$ ) arriving at a repeating pattern every year, the resulting infection level differs widely among simulations, as well as the transient: there are simulations (panel a) in which introduction of the infection results in an explosive epidemics in the first year, followed by few years in which the infection is barely detectable before reaching the stationary state; in other simulations (panel e) there is an almost steady increase of infection level to reach the stationary level. On the other hand, Figure 7 displays simulations obtained using the same set of parameters, but having set $\delta_{v}$ so as to obtain $S_{0}=0.8,1.5,2,4$. One may note that, even when $S_{0}<1$, the infection can be detected for the first 2-3 years. For values of $S_{0}>1$, after the initial outbreak, the infection decreases sharply both in mosquitoes and birds; then the disease starts increasing, converging 
Multi-year model with vertical transmission

a)

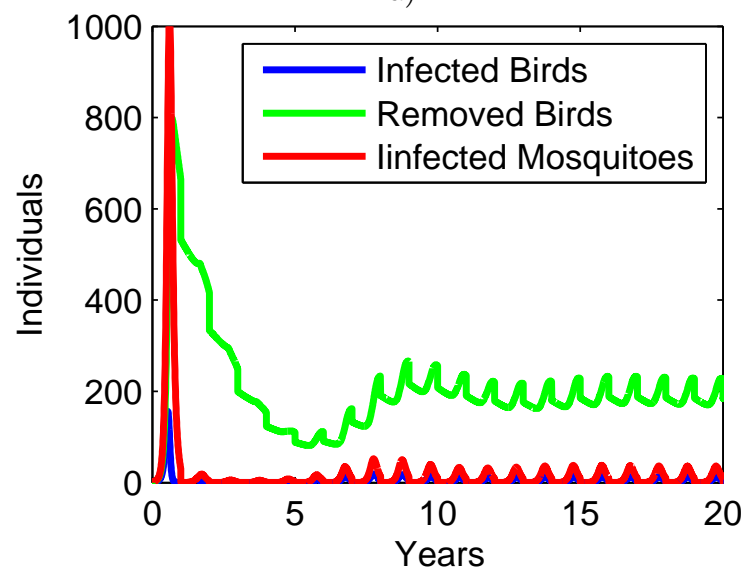

c)

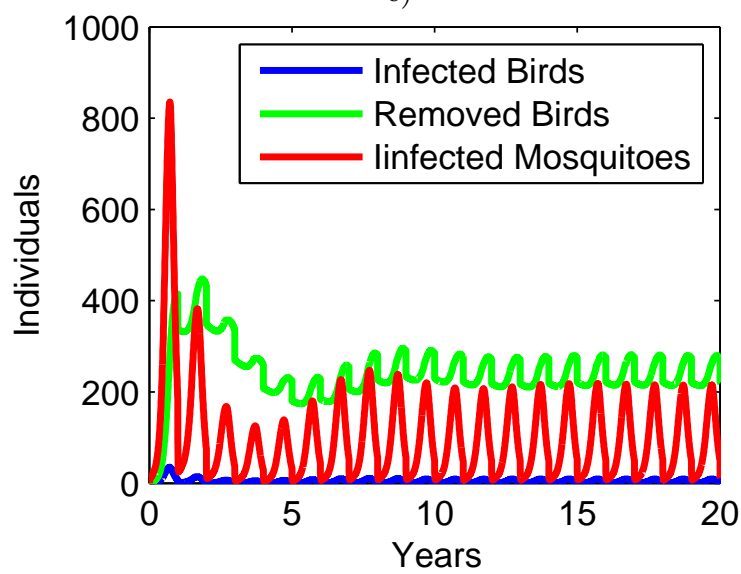

e)

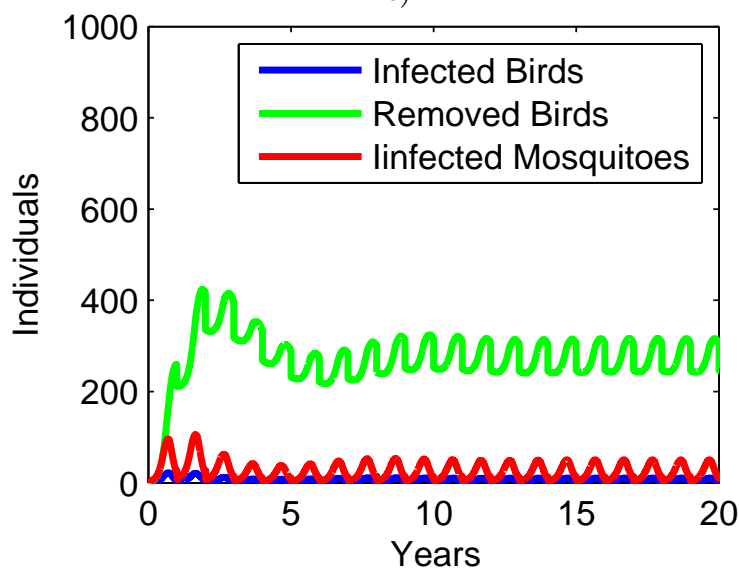

b)

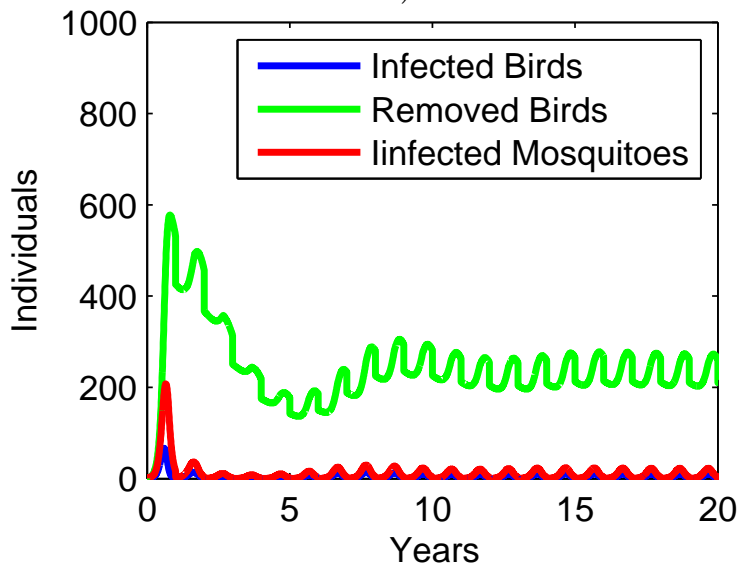

d)

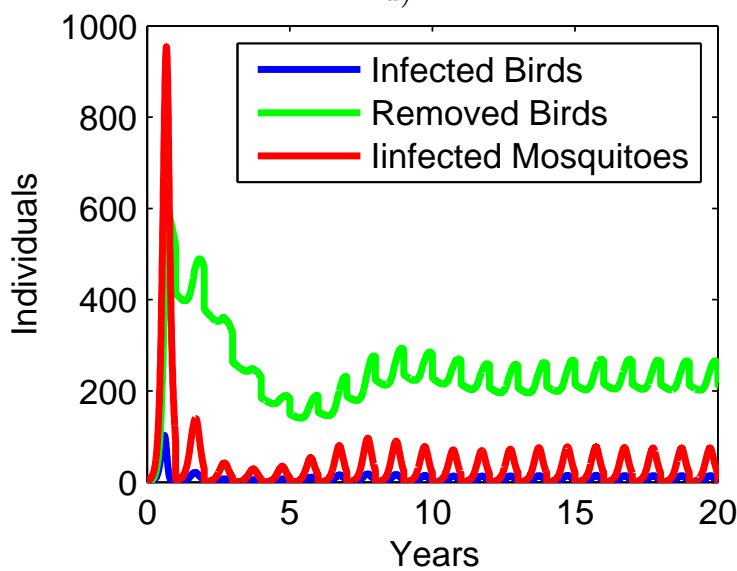

f)

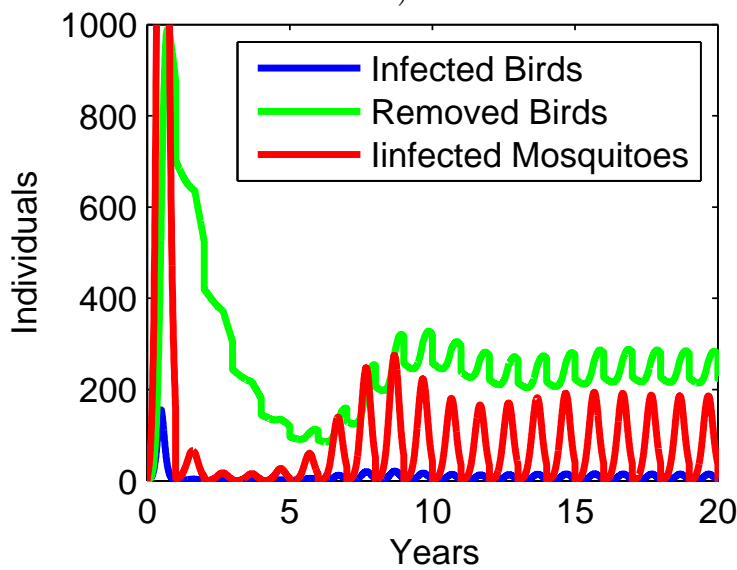

Figure 6 . Simulations of the model with vertical transmission with $S_{0}=2$. Parameter values for each panel are shown in the corresponding column of Table 2 


\begin{tabular}{|c||c|c|c|c|c|c|}
\hline Panel & $\mathrm{a})$ & $\mathrm{b})$ & $\mathrm{c})$ & $\mathrm{d})$ & $\mathrm{e})$ & $\mathrm{f})$ \\
\hline \hline$b_{2}$ & 0.1266 & 0.1266 & 0.1062 & 0.1062 & 0.1062 & 0.1062 \\
$d_{2}$ & 0.0624 & 0.06244 & 0.0549 & 0.0549 & 0.0549 & 0.0549 \\
$k_{M}$ & 91,447 & 91,447 & 193,717 & 193,717 & 193,717 & 193,717 \\
$\alpha$ & 0.6236 & 0.3852 & 0.5936 & 0.5032 & 0.5761 & 0.2618 \\
$\beta_{B}$ & 0.2428 & 0.7274 & 0.0328 & 0.1851 & 0.1006 & 0.0780 \\
$\beta_{M}$ & 0.1563 & 0.1520 & 0.5591 & 0.1655 & 0.0952 & 0.8120 \\
$\gamma$ & 0.1838 & 0.2157 & 0.2393 & 0.1606 & 0.2160 & 0.1522 \\
$\mu_{W N}$ & 0.1664 & 0.2778 & 0.1224 & 0.2309 & 0.0056 & 0.0554 \\
$\epsilon$ & 0.1463 & 0.1164 & 0.1226 & 0.1205 & 0.1495 & 0.1196 \\
$\nu$ & 0.0345 & 0.3632 & 0.0287 & 0.0359 & 0.0517 & 0.0581 \\
\hline
\end{tabular}

TABLE 2. Value of the parameters related to the plots in Figure 6

to a stationary solution that depends on the value of $S_{0}$. Still, up to values of 4 for $S_{0}$, we never observed multi-annual oscillations.

We also looked at the pattern of infection within each year; specifically, we looked at the 20th year of simulations, as a stationary situation had been reached by then. In particular we examined the peak time of infection in birds and mosquitoes, and the proportion of immune birds.

In panel a of Figure 8, we show the peak times of infected birds vs. infected mosquitoes at the 20th year. It can be seen that, for all parameter samples, the infection peak in the mosquito population occurs before, or at most simultaneously, than the peak of the bird population.

In panel $\mathrm{b}$ of Figure 8 boxplots of the density of immune birds $\left(R_{B}\right)$ are displayed for the beginning and the end of the $20^{\text {th }}$ season.

\section{Discussion}

The model examined in this work is built on a standard SIR-SIS host-vector epidemic model. There are three aspects on which it differs from more usual models for vector-borne infections: a realistic seasonal dynamics with no transmission during winter and varying population densities during each summer; consequently, explicit mechanisms of infection overwintering, for which we explored two different possibilities, both based on the assumption that the virus overwinters in infected mosquitoes; finally, a detailed description of the mosquitoes' gonotrophic cycle, resulting in only a fraction of mosquitoes actively biting at any one time.

The resulting model is rather rich in parameters. Independent estimates on most of them is scarce; hence we used the Latin Hypercube sampling scheme and rejected those samples that yielded solutions that did not satisfy some constraints. The method can be considered as a simple example of Approximate Bayesian Computation [10]: in it, parameters are rejected if the distance between simulated data and observations is above some threshold; in our case, the threshold is given by conditions (5.1)-(5.5) that refer not to specific observations but to generally accepted values.

The posterior distribution (the one obtained after rejection of samples) of most parameters is similar to the prior distribution, so that inference on parameter values is limited. However, for a few parameters, the posterior distribution is far from flat; for instance (Fig. 4), the value of $\alpha$ is likely to be in the higher proposed range (0.5-0.7), implying that the time spent searching for hosts by a mosquito is relatively short.

Another clear feature (Fig. 4d) is the highly negative correlation between the probabilities of transmission (from mosquito to bird $\beta_{B}$ and from bird to mosquito $\beta_{M}$ ): in essence the product of these probabilities can be estimated with some accuracy from field data, but not the single value of $\beta_{M}$ or $\beta_{B}$, although it is more likely (see Fig. 5) to obtain estimates with $\beta_{M}>\beta_{B}$, especially in the case of overwintering without vertical transmission. Notice that instead laboratory experiments yield a value of $\beta_{B}$ close to 1 [37], while a value of $\beta_{M}$ definitely lower [68]. The estimates shown in Fig. 5 are not in contradiction with 
Multi-year model with vertical transmission
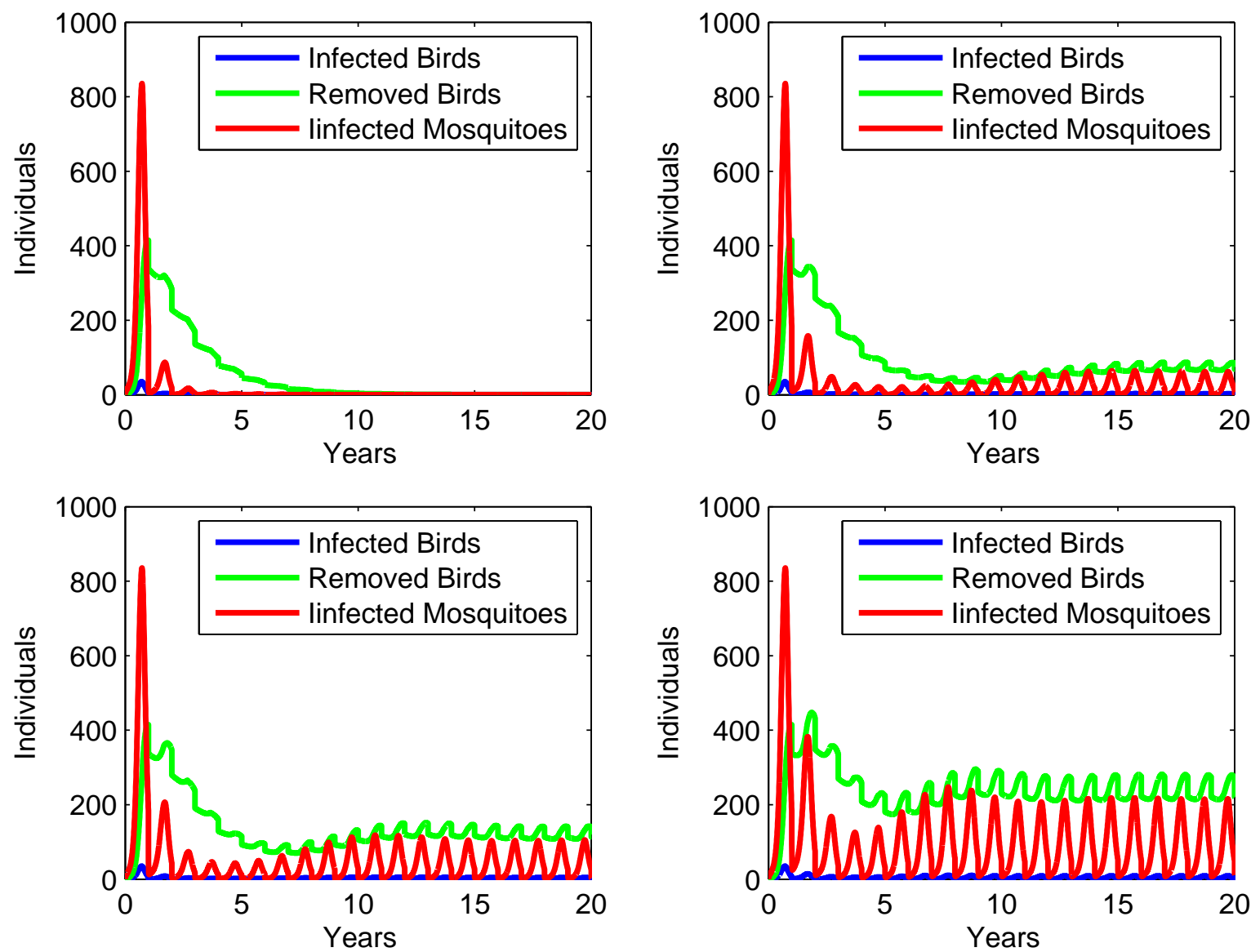

FIgURE 7. Simulations of the model with vertical transmission with with parameter values listed in column a) of Table 2) for different values of $S_{0}=0.8,1.5,2,4$

experiments, if one takes into account that mosquitoes feed on a variety of different bird species that differ widely in their infection competence [34], especially since, from the modeling point of view, infection is relevant only if it result in enough viremia to infect vectors. As the model has a single class for 'birds' on which mosquitoes feed, the resulting estimate of transmission probability is an average depending on competence of bird species, mosquito feeding preferences, and infection probability. Definitely, a more accurate model would include different bird species on which mosquitoes feed significantly, with different transmission probabilities for each of them. Moreover, a recent field study performed in US has shown that particular bird species can reduce spread of WNV (dilution effect) due to their high contact rate with mosquitoes but low competence for the virus [40]. Simpson and colleagues [66] investigated the effect of composition of bird population and mosquito feeding behavior on WNV transmission through a model considering two bird species with different competence and mosquito attractiveness. Including more than one bird species in our model would have greatly increased the number of parameters and equations. However, we preferred to adopt the current simpler model to better capture the effect of seasonality and virus overwintering on transmission dynamics of WNV.

Concerning this aspect, several mechanisms have been proposed for WNV overwintering [59], that involve winter persistence either in birds, or in mosquitoes. In the present model we considered two 

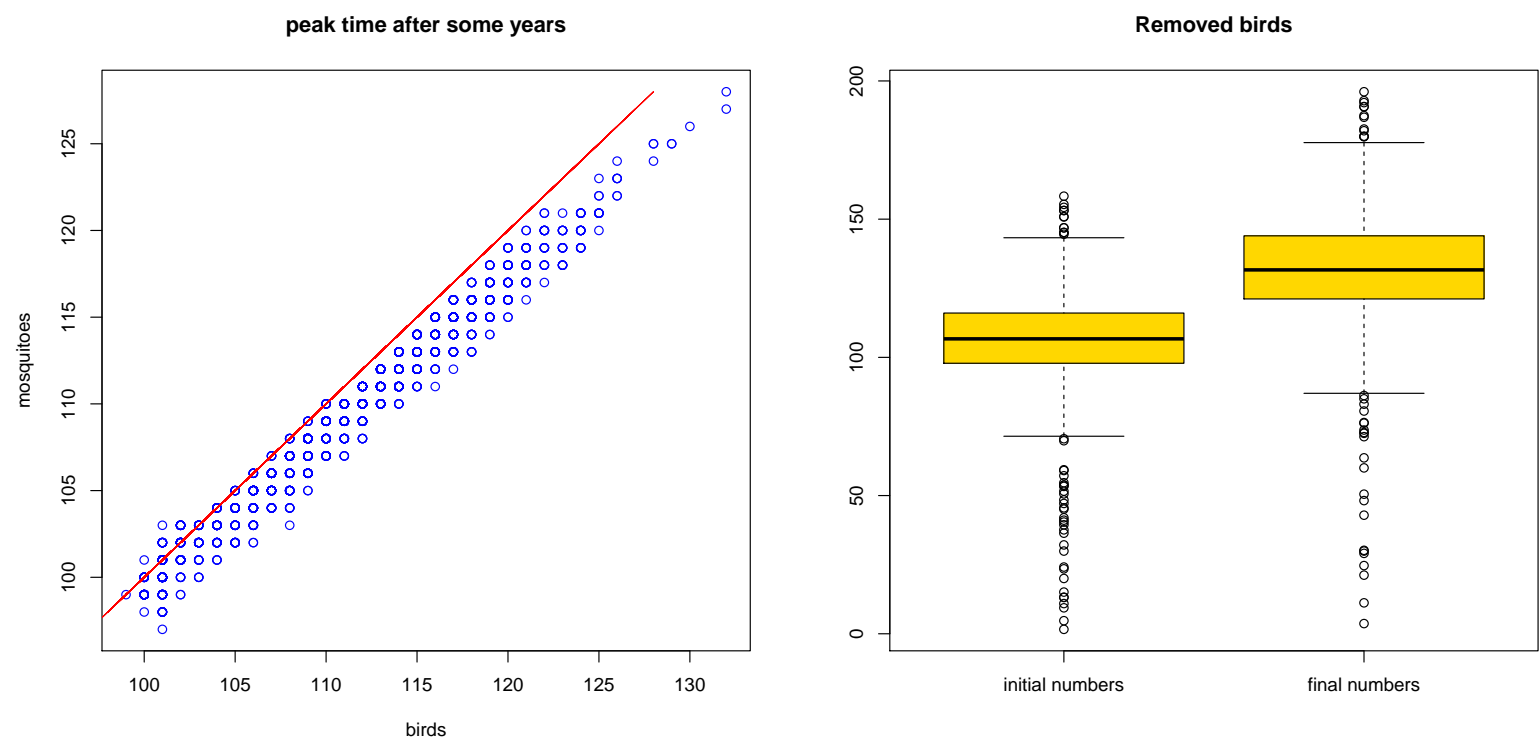

Figure 8. On the left, peak time of infected birds vs. peak time of infected mosquitoes. On the right, initial and final values of $R_{B}$ in the 20 th year of simulations. In both cases, we set $S_{0}=2$

different possibilities, both involving the mosquitoes.

The first mechanism assumes that infection is transmitted to the following year by mosquitoes that have entered diapause as unfed adults in the previous year, and must have become infected by vertical transmission [54]. The second mechanism assumes instead that a fraction of (non-diapausing) adult mosquitoes survive the winter and, if they had been infected during feeding, can transmit the infection in the following year [60].

The results obtained with either model are relatively similar; given that the model developed mainly aims at a qualitative description of the phenomena, it appears that the observed patterns may be compatible with both mechanisms. One may however note that, in the Latin Hypercube sampling it has been much easier finding parameters yielding consistent model simulations assuming overwintering of unfed diapausing adults, and vertical transmission. We can then say that this hypothesis receives some support by the current analysis, beyond the well-known evidence for survival through winter of diapausing adult adults and for vertical transmission [28].

As for overwintering mechanisms involving birds, Reisen [59] suggests that this could depend either on enzootic cycles in winter possibly involving oro-fecal transmission or on long-term infections of birds that survive the acute infection; typically, viremia in birds lasts just a few days but WNV RNA has been isolated in bird tissues up to 8 months after infection. With the present work we cannot exclude that these pathways are relevant for WNV overwintering, but we show that overwintering through diapausing females is consistent with the known features of seasonal WNV epidemics. In principle, one could also imagine that infection is brought back into Europe every spring by infected migratory birds arriving from little known endemic areas [33] but this can be definitely excluded for North America [24].

From the theoretical point of view, we defined a quantity, named $S_{0}$, that determines multi-year persistence of the virus. Intuitively, it can be defined as the expected number of infected female mosquitoes at the start of one season produced by one infected female mosquito at the start of the previous season. A similar corresponding definition could be given if overwintering occurred through infected birds, or through both. This model is a special case of periodic epidemic models analyzed by several authors 
$[6,70]$, with a part of the year with no infection transmission and through which no bird remains infected. Thus, instead of $S_{0}$, one could rely on the more traditional reproduction number $R_{0}$, whose definition and properties in the periodic case have been detailed by Bacaër and co-authors in a series of papers $[5,6]$. Indeed, $S_{0}$ and $R_{0}$ share the threshold property ( $S_{0}$ is larger, equal or smaller than 1 if and only if $R_{0}$ is larger, equal or smaller than 1 ), and $R_{0}$ has some other additional nice properties. However we think that $S_{0}$ has a more intuitive definition, can be computed easily and describes well the initial trend of infection growth from one year to the next one.

The choices used on how mosquito fertility, mortality and diapause induction depend on time within a season yield a mosquito seasonal dynamics that follows a pattern similar to the one seen in the data from [29], as shown in Figure 2. Indeed, the functions used are simply descriptive and not based on physiological mechanisms. It would be worthwhile examining the possibilities of using laboratory data (as obtained in [18] and already used for modeling population dynamics [49]) on the dependence on temperature of demographic parameters of Culex mosquitoes, coupled with an average temperature profile in the area of interest. Temperature could also have an impact on vector competence of mosquito vector species [69], a factor that should be taken in account when modeling WNV transmission in temperate regions. Possibly, other climatic factors beyond temperature are relevant for life history traits in the field (certainly diapause induction depends on photo-period), as is demonstrated in the recent analysis by [64] and in agreement with what is generally observed in reality [29]. Population dynamics of mosquito species is also affected by rainfall, thus, indirectly affecting circulation of WNV [65], and presumably resulting in different patterns at a local scale.

Outputs of model simulations are also typical infection dynamics during a season, but comparing them to actual data is difficult, as there are very limited longitudinal data. A relevant feature emerging from the simulations is that the time of the season when infected mosquitoes reach the maximum (peak-time) occurs consistently before peak-time of infected birds; this seems in agreement with field observations $[29]$.

The general multi-year pattern shows that after a large outbreak following the first introduction, a drop in cases occurs for several years, followed from an increase towards a stationary level, often with oscillations, especially if $S_{0}$ is relatively large. Such a pattern is somewhat reminiscent of the trend in human cases in United States from 1999 to 2013 [14] and reflects in an increased number of immune individuals in the bird population [38]. Recent modeling work [16] suggests that WNV is still in ascending phase in North America.

The epidemiology of WNV in Europe has been rather different, with several sporadic outbreaks and epidemic clusters often lasting a few years [9]. In the simulations with $S_{0}<1$ but close to 1, we can note (see Fig. 7) that infection may persist for 2-3 years, before the disappearance of the disease; indeed, over most of the season the instantaneous reproduction number (computed using the current values of parameters) may be larger than 1 and cause an outbreak; if overwinter survival of infected mosquitoes is such that $S_{0}$ is close to 1 , the number of survivors may be enough to start an outbreak in the following year, and perhaps even the one after. In reality, this process will be facilitated by infection spread into areas not initially affected. A similar pattern can be expected also for $S_{0}$ close to but greater than 1 , considering the possibility that the infection is eradicated because of stochastic fluctuations, when the prevalence predicted by the current deterministic model is very low.

Certainly, the model lacks biological realism in several aspects: ifirst of all, it is assumed that there is a single type of birds, while in reality Culex spp. feed on a variety of birds and mammals, some of which will be totally incompetent for WNV transmission, others will differ in their level of competence; implicitly, the model assumes that all birds on which mosquitoes feed have some average competence, which may have very different effects from assuming that birds are highly heterogeneous in competence $[34,37,66]$, because of species and age. Moreover, the model disregard changes in feeding preferences [35] that have been shown by mathematical models [50] to strongly affect infection dynamics. One can also note that bird demography has been modeled in a rather stylized way, and that no attention has been paid to mosquito larval stages in contrast to the detailed modeling of adults. Finally, we have assumed only one 
possible route for WNV over-wintering, while several mechanisms may play complementary roles.

Despite all these over-simplifications, we believe that the current model captures some important patterns resulting from the seasonal nature of mosquito-borne infections in temperate climates, and helps in understanding which overwintering mechanisms are compatible with available data. More detailed and realistic models could be built, when adequate data are available, over this backbone.

\section{A. Proof of the threshold property of $S_{0}$}

Here we compute explicitely the eigenvalues of the matrix $F^{\prime}(0,0)$ where $F$ is defined through either (4.5) or (4.6).

It is convenient to rewrite $(3.1)$ as $x^{\prime}(t)=f(t, x(t))$ where

$$
x(t)=\left(I_{B}(t), I_{M}(t), E_{M}(t), D_{I}(t), R_{B}(t), S_{B}(t), S_{M}(t), G_{M}(t)\right)
$$

and $f(t, x)$ rpresent the right hand side of (3.1), reordered in the corresponding way.

We denote $x(t ; Q, R)$ the solution of $(3.1)$ with initial conditions given by $(4.1)$ with $I_{M}(0)=Q$, $R_{B}(0)=R$. It is immediate to see that $x_{i}(t ; 0,0) \equiv 0$ for $i=1 \ldots 5$.

We can then write

$$
F^{\prime}(0,0)=\left(\begin{array}{cc}
\left.\delta_{v} \frac{\partial x_{4}(T ; Q, R)}{\partial Q}\right|_{Q=R=0} & \left.\delta_{v} \frac{\partial x_{4}(T ; Q, R)}{\partial R}\right|_{Q=R=0} \\
\left.\rho\left(\frac{\partial x_{1}(T ; Q, R)}{\partial Q}+\frac{\partial x_{5}(T ; Q, R)}{\partial Q}\right)\right|_{Q=R=0} & \left.\rho\left(\frac{\partial x_{1}(T ; Q, R)}{\partial R}+\frac{\partial x_{5}(T ; Q, R)}{\partial R}\right)\right|_{Q=R=0}
\end{array}\right)
$$

if $F$ is defined through (4.5). If $F$ is defined through (4.6), one needs only to substitute $\delta x_{2}(T ; Q, R)$ to $\delta_{v} x_{4}(T ; Q, R)$ in the first row of (A.2).

As well known, $U(t)=\nabla_{(Q, R)} x(t ; Q, R)$ is an $(8 \times 2)$ matrix that can be obtained by solving the variational system

$$
U^{\prime}(t)=\nabla_{x} f(t, x(t ; Q, R)) U(t)
$$

with initial conditions $U_{2,1}(0)=U_{5,2}(0)=1, U_{6,1}(0)=U_{7,2}(0)=-1$ and $U_{i, j}(0)=0$ for all other pairs $(i, j)$.

To compute $F^{\prime}(0,0)$, we need the matrix $U(t)$ computed for $Q=R=0$. It can be easily seen that $\frac{\partial f_{i}}{\partial x_{j}}(x(t ; 0,0))=0$ for $i \leq 5, j \geq 6$. Hence, the equations for the first 5 rows of $U(t)$ do not depend on the other ones, and we can compute

$$
u_{i}(t)=\left.\frac{\partial x_{i}(T ; Q, R)}{\partial Q}\right|_{Q=R=0} \quad v_{i}(t)=\left.\frac{\partial x_{i}(T ; Q, R)}{\partial R}\right|_{Q=R=0}, \quad i=1 \ldots 5
$$

solving the systems

$$
\begin{aligned}
& u^{\prime}(t)=A(t) u(t) \quad v^{\prime}(t)=A(t) v(t) \\
& \text { where } A(t)=\left(\begin{array}{ccccc}
-\left(\gamma+\mu_{W N}+d_{1}+Q(t) m_{1}\right) & \alpha \beta_{B} & 0 & 0 & 0 \\
0 & -(d(t)+\alpha) & f(t) \nu+\epsilon & 0 & 0 \\
\alpha \beta_{M} \frac{S_{M}(t)}{N_{B}(t)} & \alpha & -(\epsilon+d(t)) & 0 & 0 \\
0 & 0 & b(t) p(t) \nu & 0 & 0 \\
\gamma & 0 & 0 & 0 & -\left(d_{1}+Q(t) m_{1}\right)
\end{array}\right)
\end{aligned}
$$

with initial conditions

$$
u(0)=\left(\begin{array}{llllll}
0 & 1 & 0 & 0 & 0
\end{array}\right)^{T} \quad v(0)=\left(\begin{array}{lllll}
0 & 0 & 0 & 0 & 1
\end{array}\right)^{T} .
$$

In (A.3) $S_{M}(t)$ and $N_{B}(t)$ represent the solutions of (3.1) obtained with initial conditions (4.1) with $I_{M}(0)=R_{B}(0)=0$, hence they are the solutions of (2.3) and(2.1). Precisely, they solve

$$
\begin{cases}\dot{S}_{M}=b(t)(1-p(t)) G_{M}-d(t) S_{M}-\alpha S_{M}+\epsilon G_{M} & S_{M}(0)=k_{M} \\ \dot{G}_{M}=\alpha S_{M}-\epsilon G_{M}-d(t) G_{M} & G_{M}(0)=0 \\ \dot{N}_{B}=P b_{1} N_{B}-d_{1} N_{B}-Q(t) m_{1} N_{B} & N_{B}(0)=k_{B}\end{cases}
$$


From the structure of matrix $A$, one immediately sees that $v_{i}(t) \equiv 0$ for $i=1 \ldots 4$. Hence, from (A.2), one sees that $\left(F^{\prime}(0,0)\right)_{1,2}=0$; the eigenvalues of $F^{\prime}(0,0)$ are then its diagonal elements. Notice that the result is the same if $F$ were given by (4.6).

Furthermore from (A.3)-(A.4), one obtains

$$
\left(F^{\prime}(0,0)\right)_{2,2}=\rho v_{5}(T)=\rho e^{-d_{1} T-m_{1} \int_{0}^{T} Q(t) d t}<1 .
$$

Hence $\rho\left(F^{\prime}(0,0)\right)<1$ if and only if $S_{0}<1$, where $S_{0}$ is defined as $S_{0}=\left(F^{\prime}(0,0)\right)_{1,1}$. We then have $S_{0}=\delta_{v} u_{4}(T)$ or $S_{0}=\delta u_{2}(T)$ according to whether $F$ is defined through (4.5) or through (4.6). This is exactly the definition given in (4.8) or (4.9) of the main text.

\section{B. Model with immature stages}

Since immature stages of mosquitoes do not contribute to infection transmission, the simplest method to include immature stages of mosquitoes in the models considered in the main text is to assume there is a delay $\tau$ between the time of egg laying, and that of adult emergence.

Thus, system (2.3) becomes

$$
\left\{\begin{array}{l}
\dot{F}(t)=b(t-\tau)(1-p(t-\tau)) \sigma G(t-\tau)-d(t) F(t)-\alpha F(t)+\epsilon G(t) \\
\dot{G}(t)=\alpha F(t)-\epsilon G(t)-d(t) G(t) .
\end{array}\right.
$$

The rate at which new mosquitoes enter the host-seeking adult population at time $t$ depends on fertility $b(t-\tau)$ of mosquitoes in the gonotrophic stage $G(t-\tau) \tau$ time before, times the probability $1-p(t-\tau)$ of not producing diapausing adults, times the probability $\sigma$ of surviving through the juvenile stages.

Similarly, the main model (3.1) would be changed to

$$
\left\{\begin{array}{l}
\dot{S}_{B}(t)=P(t) b_{1} N_{B}(t)-d_{1} S_{B}(t)-\alpha \frac{\beta_{B} I_{M}(t)}{N_{B}(t)} S_{B}(t)-Q(t) m_{1} S_{B}(t) \\
\dot{I}_{B}(t)=\alpha \frac{\beta_{B} I_{M}(t)}{N_{B}(t)} S_{B}(t)-\gamma I_{B}(t)-\mu_{W N} I_{B}(t)-d_{1} I_{B}(t)-Q(t) m_{1} I_{B}(t) \\
\dot{R}_{B}(t)=\gamma I_{B}(t)-d_{1} R_{B}(t)-Q m_{1} R_{B}(t) \\
\dot{S}_{M}(t)=b(t-\tau)(1-p(t-\tau)) \sigma\left((1-\nu) E_{M}(t-\tau)+G_{M}(t-\tau)\right)-d(t) S_{M}(t)-\alpha S_{M}(t)+\epsilon G_{M}(t) \\
\dot{E}_{M}(t)=\alpha \frac{\beta_{M} I_{B}(t)}{N_{B}(t)} S_{M}(t)-\epsilon E_{M}(t)-d(t) E_{M}(t)+\alpha I_{M}(t) \\
\dot{G}_{M}(t)=\alpha \frac{S_{B}(t)+R_{B}(t)+\left(1-\beta_{M}\right) I_{B}(t)}{N_{B}(t)} S_{M}(t)-\epsilon G_{M}(t)-d(t) G_{M}(t) \\
\dot{I}_{M}(t)=b(t-\tau)(1-p(t-\tau)) \sigma \nu E_{M}(t-\tau)+\epsilon E_{M}(t)-d(t) I_{M}(t)-\alpha I_{M}(t) \\
\dot{D}_{I}(t)=b(t-\tau) p(t-\tau) \sigma \nu E_{M}(t-\tau) .
\end{array}\right.
$$

A deficiency in the above models is that the delay between egg-laying and adult emergence is fixed, while it is well known that developmental times strongly depend on temperature and other environmental features [43]. This problem could be handled by allowing for variable developmental times, although this leads to more complex models [26]. Alternatively, one can explicitly model the population dynamics of several immature stages (eggs, different instar larvae, pupae) with temperature-dependent rates of moving to the next stage [49].

Another useful improvement of the models would be considering bird age-structure, or at least distinguishing between hatch-year and older birds, as it has been shown [29] that the former play an important role in WNV dynamics. This will be the focus of future work. 
Multi-year model without vertical transmission

a)

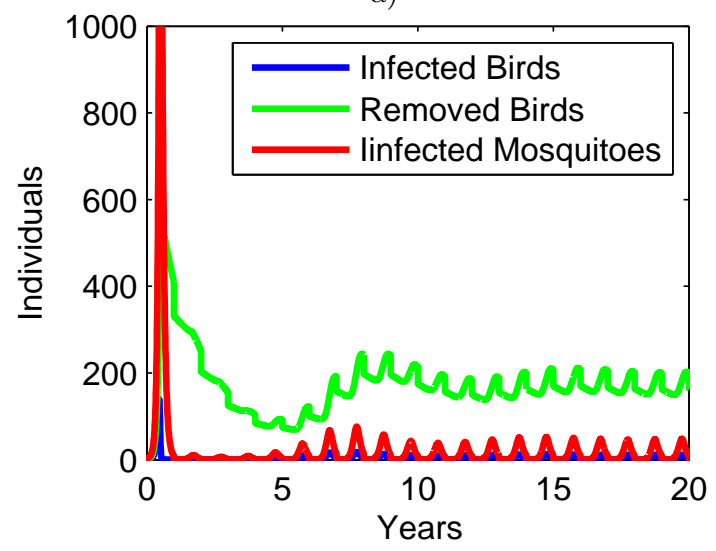

c)

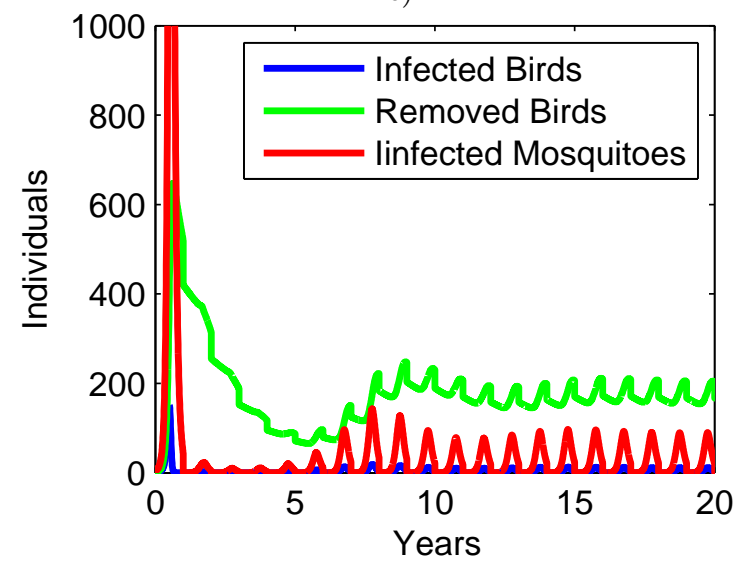

e)

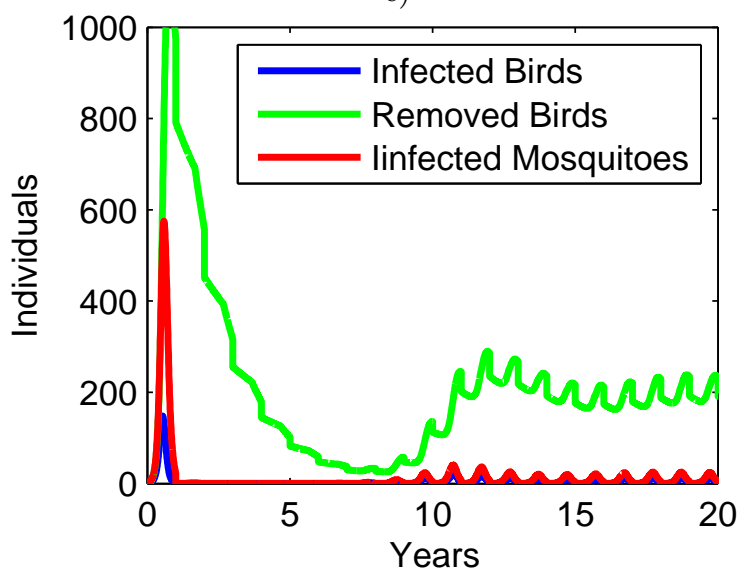

b)

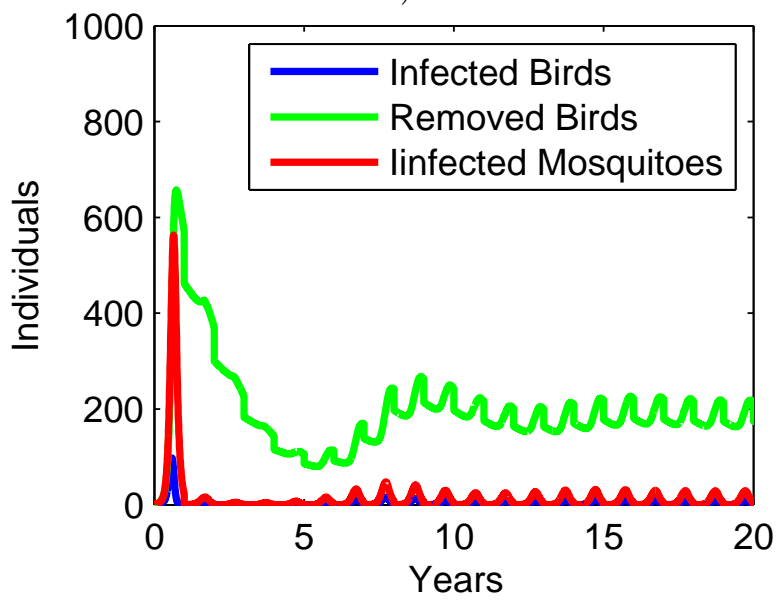

d)

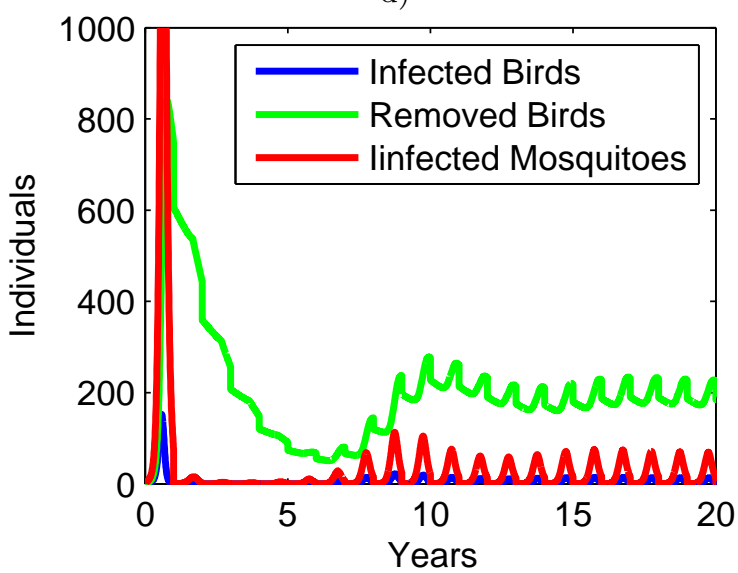

f)

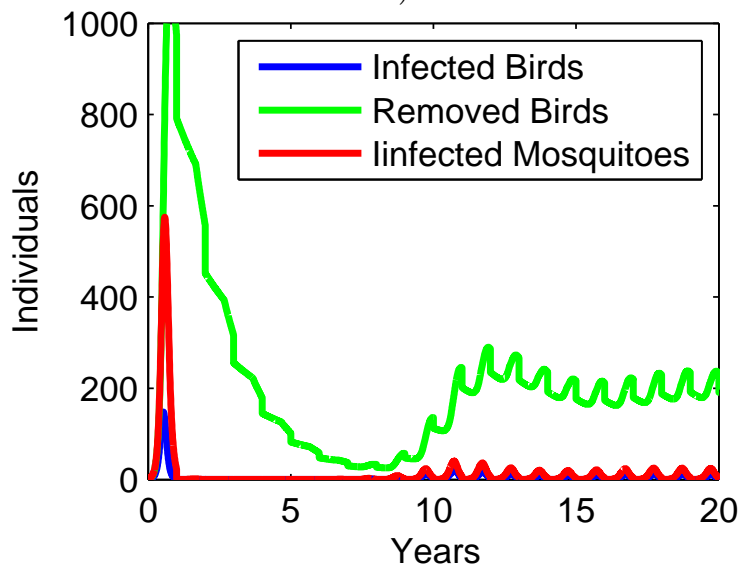

Figure C.1. Simulations of the model without vertical transmission with $S_{0}=2$. Parameter values for each panel are shown in the corresponding column of Table C.1. 


\begin{tabular}{|c||c|c|c|c|c|c|}
\hline Panel & a) & b) & c) & d) & e) & f) \\
\hline \hline$b_{2}$ & 0.1266 & 0.1266 & 0.1062 & 0.1062 & 0.1062 & 0.1062 \\
$d_{2}$ & 0.0624 & 0.06244 & 0.0549 & 0.0549 & 0.0549 & 0.0549 \\
$k_{M}$ & 91,447 & 91,447 & 91,447 & 193,717 & 193,717 & 193,717 \\
$\alpha$ & 0.5187 & 0.5132 & 0.5562 & 0.3071 & 0.5827 & 0.6891 \\
$\beta_{B}$ & 0.1013 & 0.1769 & 0.0876 & 0.0992 & 0.0606 & 0.1110 \\
$\beta_{M}$ & 0.6621 & 0.3181 & 0.3399 & 0.9991 & 0.5784 & 0.1112 \\
$\gamma$ & 0.1981 & 0.2334 & 0.1543 & 0.1605 & 0.1982 & 0.2174 \\
$\mu_{W N}$ & 0.3476 & 0.3622 & 0.0478 & 0.4646 & 0.3548 & 0.1249 \\
$\epsilon$ & 0.1203 & 0.1340 & 0.1346 & 0.1438 & 0.1327 & 0.1175 \\
\hline
\end{tabular}

TABLE C.1. Value of the parameters related to the plots in Figure C.1

\section{Simulations of the model without vertical transmission}

The results regarding the model without vertical transmission are displayed in the following Figures.

The results are qualitatively quite similar to the case with vertical transmission.

Figures C.2 show how the disease reaches a stable point more slowly than in the case of the model with vertical transmission.
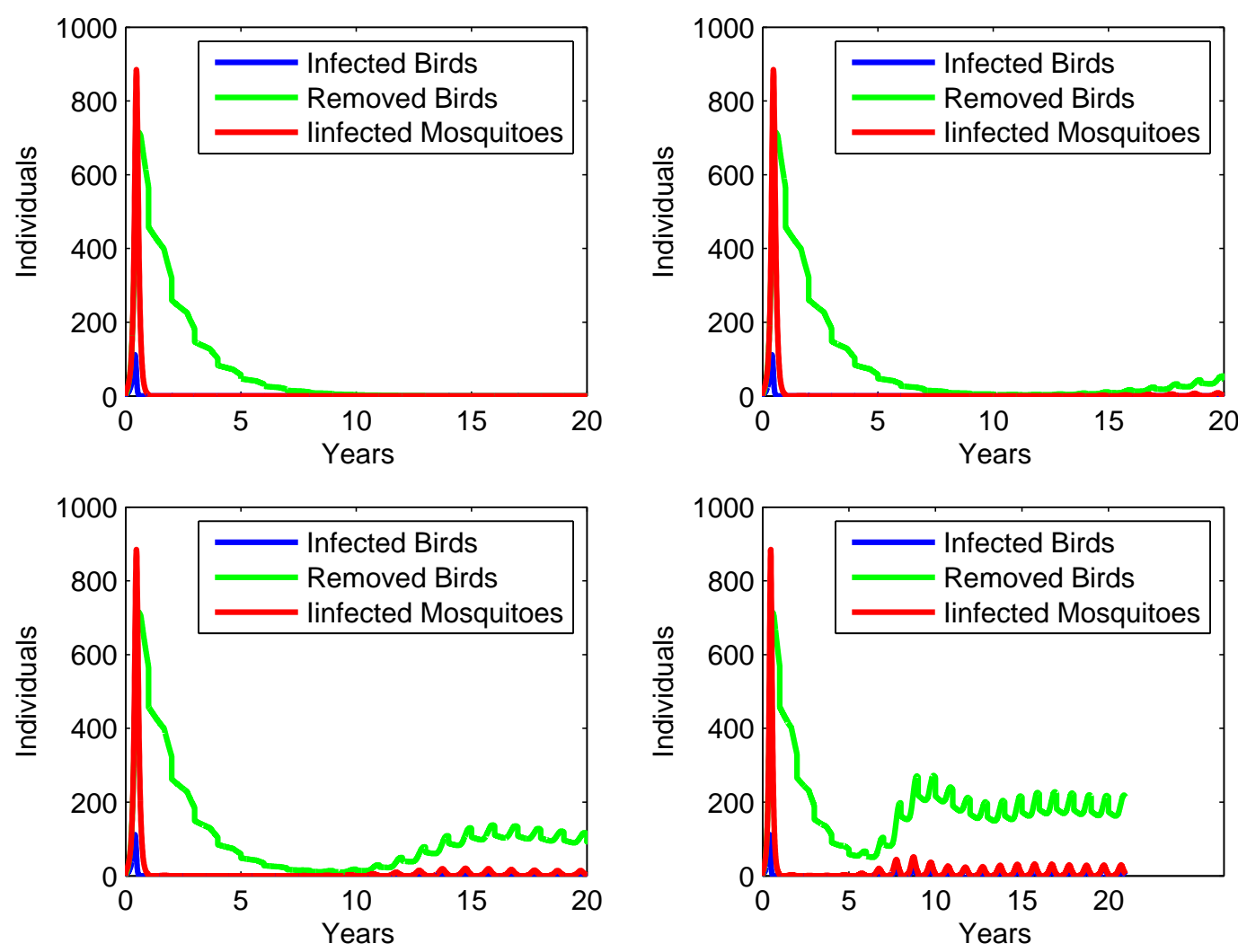

Figure C.2. Simulations of the model without vertical transmission with parameter values listed in column a) of Table C. 1 for values of $S_{0}=0.2,1.5,2,4$ 

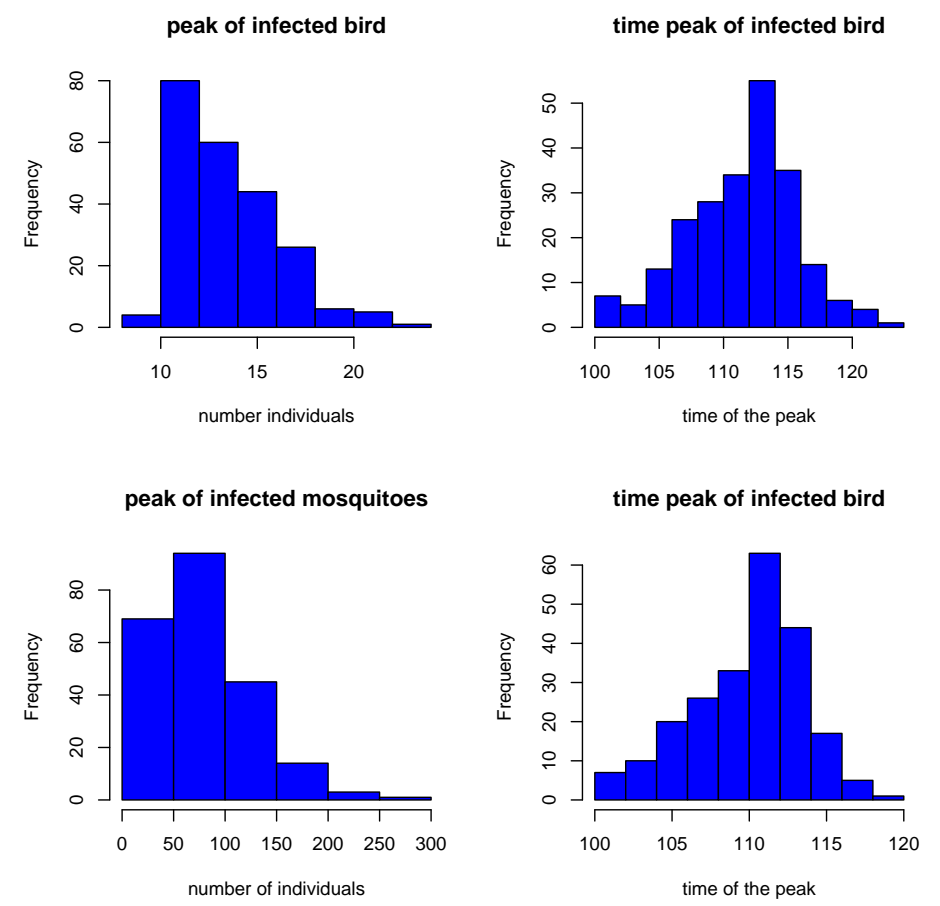

Figure C.3. Peaks and times of peak of infected birds and mosquitoes (Model without vertical transmission)

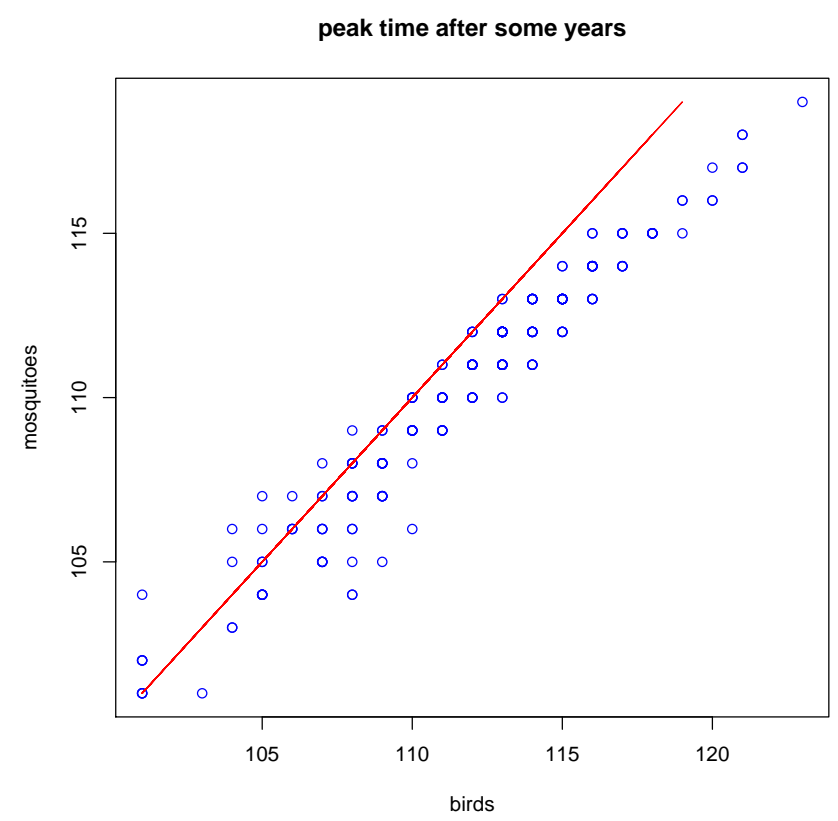

Figure C.4. Peak time of infected bird versus peak time of infected mosquitoes 

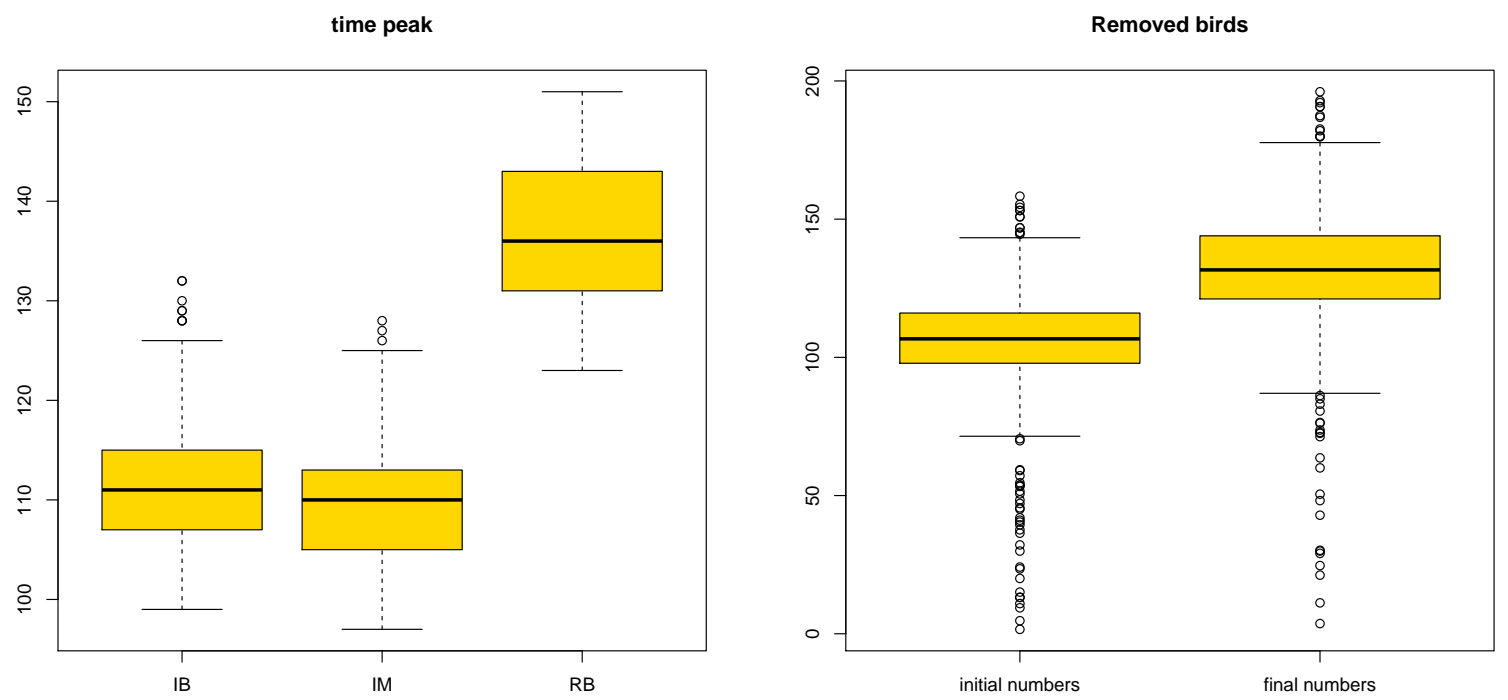

Figure C.5. Left panel: Peak time of infected bird, peak time of infected mosquitoes and peak time of removed birds. Right panel: Initial and final $R_{B}$ at $20^{\text {th }}$ in the model without vertical transmission

\section{Simulations of $20^{\text {th }}$ year}
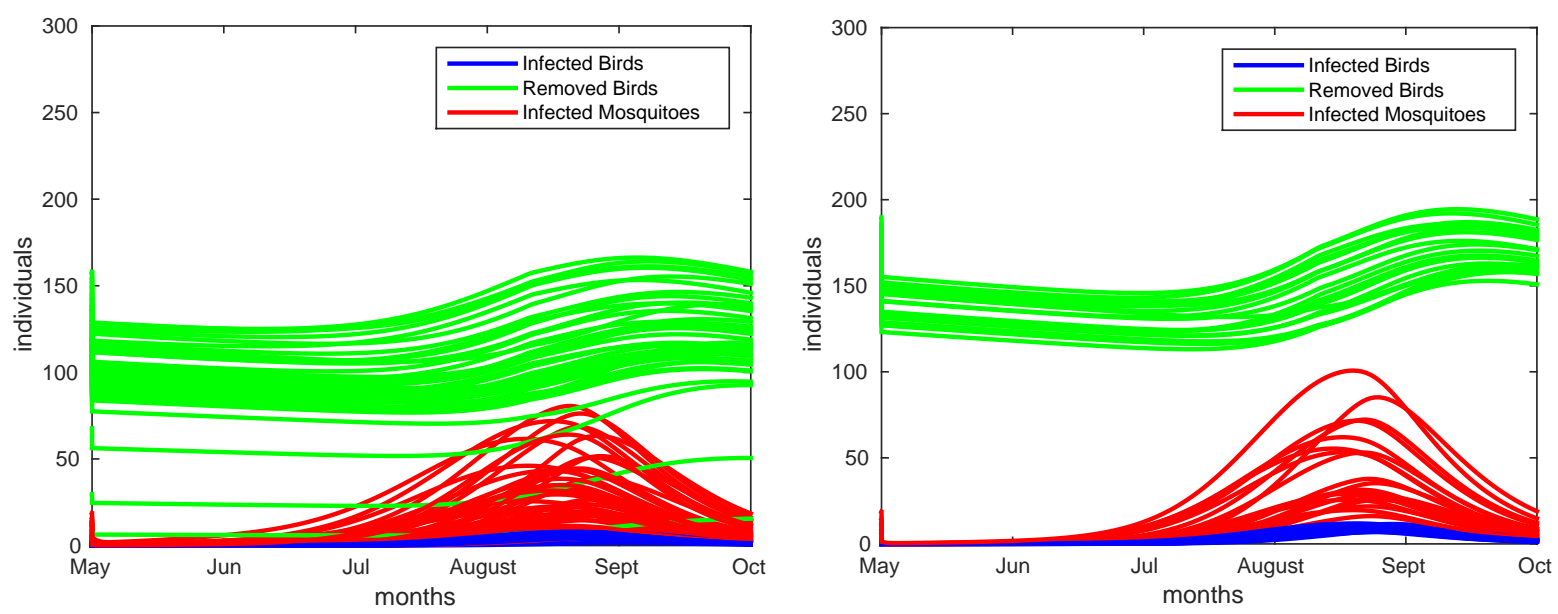

Figure C.6. Plot of the $20^{\text {th }}$ year of some simulations starting with only one infected bird at the start of year 0 . Left panel: $S_{0}=2$; right panel: $S_{0}=3$. In red infected mosquitoes, in blue infected birds and in green removed birds. 
Acknowledgements. We wish to thank Uriel Kitron and Gonzalo Vazquez-Prokopec for providing many useful suggestions on model development. We also thank two anonymus referees for their useful comments and suggestions. AP was partially supported through Project LExEM (Laboratory of excellence for epidemiology and modelling, http://www.lexem.eu), Research funds for Grandi Progetti, Autonomous Province of Trento (Italy).

\section{References}

[1] A. Abdelrazec, S. Lenhart, H. Zhu. Transmission dynamics of West Nile virus in mosquitoes and corvids and noncorvids. J. Math. Biol., 68(6):1553-82, 2014.

[2] R. M. Anderson, R. M. May. Infectious diseases of humans, volume 1. Oxford university press Oxford, 1991.

[3] T. G. Andreadis, J. F. Anderson, C. R. Vossbrinck, A. J. Main. Epidemiology of West Nile virus in Connecticut: a five-year analysis of mosquito data 1999-2003. Vector-Borne \& Zoonotic Diseases, 4(4):360-378, 2004.

[4] C. S. Apperson, H. K. Hassan, B. A. Harrison, H. M. Savage, S. E. Aspen, A. Farajollahi, W. Crans, T. J. Daniels, R. C. Falco, M. Benedict, et al. Host feeding patterns of established and potential mosquito vectors of West Nile virus in the Eastern United States. Vector-Borne and Zoonotic Diseases, 4(1):71-82, 2004.

[5] N. Bacaër. Approximation of the basic reproduction number $R_{0}$ for vector-borne diseases with a periodic vector population. Bulletin of mathematical biology, 69(3):1067-1091, 2007.

[6] N. Bacaër, S. Guernaoui. The epidemic threshold of vector-borne diseases with seasonality. Journal of mathematical biology, 53(3):421-436, 2006.

[7] C. L. Bailey, M. E. Faran, T. Gargan 2nd, D. E. Hayes. Winter survival of blood-fed and nonblood-fed Culex pipiens L. The American journal of tropical medicine and hygiene, 31(5):1054-1061, 1982.

[8] S. Baqar, C. G. Hayes, J. R. Murphy, D. M. Watts. Vertical transmission of West Nile virus by Culex and Aedes species mosquitoes. Technical report, DTIC Document, 1993.

[9] L. Barzon, M. Pacenti, E. Franchin, L. Squarzon, E. Lavezzo, M. Cattai, R. Cusinato, G. Palù. The complex epidemiological scenario of West Nile virus in Italy. Int. J. Environ. Res. Public Health, 10(10):4669-4689, 2013.

[10] M. Beaumont. Approximate Bayesian computation in evolution and ecology. Annual Review of Ecology, Evolution, and Systematics, 41:379-406, 2010

[11] D. Bisanzio, M. Giacobini, L. Bertolotti, A. Mosca, L. Balbo, U. Kitron, G. M. Vazquez-Prokopec. Spatio-temporal patterns of distribution of West Nile virus vectors in eastern Piedmont Region, Italy. Parasit Vectors, 4:230, 2011.

[12] C. Bowman, A. B. Gumel, P. Van Den Driessche, J. Wu, H. Zhu. A mathematical model for assessing control strategies against West Nile virus. Bull. Math. Biol., 67(5):1107-1133, 2005.

[13] J. Castillo-Olivares, J. Wood. West Nile virus infection of horses. Veterinary research, 35(4):467-483, 2004.

[14] CDC. www.cdc.gov/westnile/. Centers of Disease Control and Prevention, 2014.

[15] CDC. $h$ ttp://www.cdc.gov/westnile/statsmaps/. Centers of Disease Control and Prevention, september 2013.

[16] J. Chen, J. Huang, J. C. Beier, R. S. Cantrell, C. Cosner, D. O. Fuller, G. Zhang, S. Ruan. Modeling and control of local outbreaks of West Nile virus in the United States. Discret. Contin. Dyn. Syst. - Ser. B, 21(8):2423-2449, 2016.

[17] D. Cianci, J. Van Den Broek, B. Caputo, F. Marini, A. D. Torre, H. Heesterbeek, N. Hartemink. Estimating Mosquito Population Size From Mark?Release?Recapture Data. J. Med. Entomol., 50(3):533, 2014.

[18] A. T. Ciota, A. C. Matacchiero, A. M. Kilpatrick, L. D. Kramer. The effect of temperature on life history traits of Culex mosquitoes. Journal of medical entomology, 51(1):55-62, 2014.

[19] G. Cruz-Pacheco, L. Esteva, J. A. Montaõ-Hirose, C. Vargas. Modelling the dynamics of West Nile virus. Bulletin of mathematical biology, 67(6):1157-1172, 2005.

[20] G. Cruz-Pacheco, L. Esteva, C. Vargas. Seasonality and outbreaks in West Nile virus infection. Bull. Math. Biol., 71(6):1378-93, 2009.

[21] G. Cruz-Pacheco, L. Esteva, C. Vargas. Multi-species interactions in West Nile virus infection. J. Biol. Dyn., 6(2):281$298,2012$.

[22] J. R. Dawson, W. B. Stone, G. D. Ebel, D. S. Young, D. S. Galinski, J. P. Pensabene, M. A. Franke, M. Eidson, L. D. Kramer. Crow deaths caused by West Nile virus during winter. Emerging infectious diseases, 13(12):1912, 2007.

[23] B. L. Dodson, L. D. Kramer, J. L. Rasgon. Effects of larval rearing temperature on immature development and West Nile virus vector competence of Culex tarsalis. Parasit. Vectors, 5(1):1-6, 2012.

[24] N. K. Duggal, W. K. Reisen, Y. Fang, R. M. Newman, X. Yang, G. D. Ebel, A. C. Brault. Genotype-specific variation in West Nile virus dispersal in California. Virology, 485:79-85, 2015.

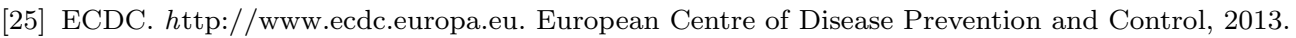

[26] D. A. Ewing, C. A. Cobbold, B. V. Purse, M. A. Nunn, S. M. White. Modelling the effect of temperature on the seasonal population dynamics of temperate mosquitoes. J. Theor. Biol., 400:65-79, 2016.

[27] C. Faraj, M. Elkohli, M. Lyagoubi. Cycle gonotrophique de Culex pipiens (Diptera : Culicidae), vecteur potentiel du virus West Nile, au Maroc : estimation de la durée en laboratoire. Bull Soc Pathol Exot, 99:119-121, 2006.

[28] L. B. Goddard, A. E. Roth, W. K. Reisen, T. W. Scott. Vertical transmission of West Nile virus by three California Culex (Diptera: Culicidae) species. Journal of medical entomology, 40(6):743-746, 2003.

[29] G. L. Hamer, E. D. Walker, J. D. Brawn, S. R. Loss, M. O. Ruiz, T. L. Goldberg, A. M. Schotthoefer, W. M. Brown, E. Wheeler, U. D. Kitron. Rapid amplification of West Nile virus: the role of hatch-year birds. Vector-Borne and Zoonotic Diseases, 8(1):57-68, 2008. 
[30] E. B. Hayes, N. Komar, R. S. Nasci, S. P. Montgomery, D. R. O'Leary, G. L. Campbell, et al. Epidemiology and transmission dynamics of West Nile virus disease. Emerg Infect Dis, 11(8):1167-1173, 2005.

[31] Z. Hubálek, J. Halouzka. West Nile fever-a reemerging mosquito-borne viral disease in europe. Emerging infectious diseases, 5(5):643, 1999.

[32] J. Jiang, Z. Qiu, J. Wu, H. Zhu. Threshold conditions for West Nile virus outbreaks. Bull. Math. Biol., 71(3):627-47, 2009.

[33] E. Jourdain, H. G. Zeller, P. Sabatier, S. Murri, Y. Kayser, T. Greenland, M. Lafaye, M. Gauthier-Clerc. Prevalence of West Nile virus neutralizing antibodies in wild birds from the Camargue area, southern France. J. Wildl. Dis., 44(3):766-771, 2008.

[34] A. M. Kilpatrick, P. Daszak, M. J. Jones, P. P. Marra, L. D. Kramer. Host heterogeneity dominates West Nile virus transmission. Proceedings of the Royal Society B: Biological Sciences, 273(1599):2327-2333, 2006.

[35] A. M. Kilpatrick, L. D. Kramer, M. J. Jones, P. P. Marra, P. Daszak. West Nile virus epidemics in North America are driven by shifts in mosquito feeding behaviour. Plos Biology, 4, 2006.

[36] N. Komar. West Nile virus: epidemiology and ecology in North America. Advances in virus research, 61:185-234, 2003.

[37] N. Komar, S. Langevin, S. Hinten, N. M. Nemeth, E. Edwards, D. L. Hettler, B. S. Davis, R. A. Bowen, M. L. Bunning. Experimental Infection of North American Birds with the New York 1999 Strain of West Nile Virus. Emerg. Infect. Dis. J., 9(3):311, 2003

[38] J. L. Kwan, S. Kluh, W. K. Reisen. Antecedent avian immunity limits tangential transmission of West Nile virus to humans. PLoS One, 7(3):e34127, 2012.

[39] V. Laperriere, K. Brugger, F. Rubel. Simulation of the seasonal cycles of bird, equine and human West Nile virus cases. Preventive veterinary medicine, 98(2):99-110, 2011.

[40] R. S. Levine, D. G. Mead, G. L. Hamer, B. J. Brosi, D. L. Hedeen, M. W. Hedeen, J. R. McMillan, D. Bisanzio, U. D. Kitron. Supersuppression: Reservoir competency and timing of mosquito host shifts combine to reduce spillover of West Nile virus. The American Journal of Tropical Medicine and Hygiene, pages 15-0809, 2016.

[41] M. Lewis, J. Rencławowicz, P. van den Driessche. Traveling waves and spread rates for a West Nile virus model. Bull. Math. Biol., 68(1):3-23, 2006.

[42] R. Liu, J. Shuai, J. Wu, H. Zhu. Modeling spatial spread of west nile virus and impact of directional dispersal of birds. Math. Biosci. Eng., 3(1):145-160, 2006.

[43] V. Loetti, N. Schweigmann, N. Burroni. Development rates, larval survivorship and wing length of Culex pipiens (Diptera: Culicidae) at constant temperatures. J. Nat. Hist., 45(35-36):2203-2213, 2011.

[44] G. Macdonald et al. The epidemiology and control of malaria. The Epidemiology and Control of Malaria., 1957.

[45] D. J. Madder, G. A. Surgeoner, B. V. Helson. Number of generations, egg production, and developmental time of Culex pipiens and Culex restauns (Diptera: Culicidae) in southern Ontario. J Med Entomol, 20, 1983.

[46] N. a. Maidana, H. M. Yang. Spatial spreading of West Nile Virus described by traveling waves. J. Theor. Biol., 258(3):403-417, 2009.

[47] T. Malik, P. Salceanu, A. Mubayi, A. Tridane, M. Imran. West Nile dynamics : virus transmission between domestic and wild bird populations through vectors. Can. Appl. Math. Quart., 20(4):535-556, 2012.

[48] A. Mannelli, M. Martello, L. Tomassone, M. Calzolari, C. Casalone, et al. Inventory of available data and data sources and proposal for data collection on vector-borne zoonoses in animals. EFSA External Scientific Report en-234, 2012.

[49] G. Marini, P. Poletti, M. Giacobini, A. Pugliese, S. Merler, R. Rosà. The Role of Climatic and Density Dependent Factors in Shaping Mosquito Population Dynamics: The Case of Culex pipiens in Northwestern Italy. PLoS One, 11(4):e0154018, apr 2016.

[50] G. Marini, R. Rosá, A. Pugliese, H. Heesterbeek. Exploring vector-borne infection ecology in multi-host communities: A case study of West Nile virus. J. Theor. Biol., 415:58-69, 2017.

[51] S. Marino, I. B. Hogue, C. J. Ray, D. E. Kirschner. A methodology for performing global uncertainty and sensitivity analysis in systems biology. Journal of theoretical biology, 254(1):178-196, 2008.

[52] G. Molaei, T. G. Andreadis, P. M. Armstrong, J. F. Anderson, C. R. Vossbrinck. Host feeding patterns of Culex mosquitoes and West Nile virus transmission, northeastern United States. Emerging infectious diseases, 12(3):468, 2006 .

[53] R. S. Nasci, H. M. Savage, D. J. White, J. R. Miller, B. C. Cropp, M. S. Godsey, A. J. Kerst, P. Bennett, K. Gottfried, R. S. Lanciotti. West Nile virus in overwintering Culex mosquitoes, New York City, 2000. Emerging Infectious Diseases, 7(4):742, 2001.

[54] B. M. Nelms, E. Fechter-Leggett, B. D. Carroll, P. Macedo, S. Kluh, W. K. Reisen. Experimental and natural vertical transmission of West Nile virus by California Culex (Diptera: Culicidae) mosquitoes. Journal of medical entomology, 50(2):371-378, 2013.

[55] B. R. Noon, J. R. Sauer. Population models for passerine birds: structure, parameterization, and analysis. In Wildlife 2001: populations, pages 441-464. Springer, 1992.

[56] J. Owen, F. R. Moore, A. Williams, M. Ward, T. Beveroth, E. Miller, L. Wilson, V. Morley, R. Abbey-Lee, B. Veeneman, et al. Test of recrudescence hypothesis for overwintering of West Nile virus in gray catbirds. Journal of medical entomology, 47(3):451-457, 2010.

[57] E. Pérez-Ramírez, F. Llorente, M. Á. Jiménez-Clavero. Experimental Infections of Wild Birds with West Nile Virus. Viruses, 6(2):752-781, feb 2014.

[58] E. A. E. Rayah, N. A. A. Groun. Effect of temperature on hatching eggs and embryonic survival in the mosquito Culex quinquefasciatus. Entomol. Exp. Appl., 33(3):349-351, 1983. 
[59] W. K. Reisen. Ecology of West Nile virus in North America. Viruses, 5(9):2079-2105, 2013.

[60] W. K. Reisen, Y. Fang, H. D. Lothrop, V. M. Martinez, J. Wilson, P. O'Connor, R. Carney, B. Cahoon-Young, M. Shafii, A. C. Brault. Overwintering of West Nile Virus in Southern California. J. Med. Entomol., 43(2):344-355, 2006.

[61] W. K. Reisen, Y. Fang, V. M. Martinez. Effects of Temperature on the Transmission of West Nile Virus by Culex tarsalis (Diptera: Culicidae). J. Med. Entomol., 43(2):309-317, mar 2006.

[62] P. Reiter. Climate change and mosquito-borne disease. Environmental health perspectives, 109(Suppl 1):141, 2001.

[63] A. Rizzoli, L. Bolzoni, E. a. Chadwick, G. Capelli, F. Montarsi, M. Grisenti, J. M. de la Puente, J. Muñoz, J. Figuerola, R. Soriguer, G. Anfora, M. Di Luca, R. Rosà. Understanding West Nile virus ecology in Europe: Culex pipiens host feeding preference in a hotspot of virus emergence. Parasit. Vectors, 8(1):213, 2015.

[64] R. Rosà, G. Marini, L. Bolzoni, M. Neteler, M. Metz, L. Delucchi, E. A. Chadwick, L. Balbo, A. Mosca, M. Giacobini, et al. Early warning of West Nile virus mosquito vector: climate and land use models successfully explain phenology and abundance of Culex pipiens mosquitoes in north-western Italy. Parasites \& vectors, 7(1):269, 2014.

[65] L. Shand, W. M. Brown, L. F. Chaves, T. L. Goldberg, G. L. Hamer, L. Haramis, U. Kitron, E. D. Walker, M. O. Ruiz. Predicting West Nile virus infection risk from the synergistic effects of rainfall and temperature. Journal of medical entomology, page tjw042, 2016.

[66] J. E. Simpson, P. J. Hurtado, J. Medlock, G. Molaei, T. G. Andreadis, A. P. Galvani, M. A. Diuk-Wasser. Vector host-feeding preferences drive transmission of multi-host pathogens: West Nile virus as a model system. Proceedings of the Royal Society B: Biological Sciences, 279(1730):925-933, 2012.

[67] D. Thomas, B. Urena. A model describing the evolution of West Nile-like encephalitis in New York city. Mathematical and computer modelling, 34(7):771-781, 2001.

[68] M. J. Turell, D. J. Dohm, M. R. Sardelis, M. L. O'guinn, T. G. Andreadis, J. A. Blow. An update on the potential of north american mosquitoes (diptera: Culicidae) to transmit West Nile virus. Journal of medical entomology, 42(1):57$62,2005$.

[69] C. B. Vogels, J. J. Fros, G. P. Göertz, G. P. Pijlman, C. J. Koenraadt. Vector competence of northern European Culex pipiens biotypes and hybrids for West Nile virus is differentially affected by temperature. Parasites \& Vectors, 9(1):393, 2016.

[70] W. Wang, X.-Q. Zhao. Threshold dynamics for compartmental epidemic models in periodic environments. Journal of Dynamics and Differential Equations, 20(3):699-717, 2008.

[71] S. S. Wheeler, S. A. Langevin, A. C. Brault, L. Woods, B. D. Carroll, W. K. Reisen. Detection of persistent West Nile virus RNA in experimentally and naturally infected avian hosts. The American journal of tropical medicine and hygiene, 87(3):559-564, 2012.

[72] M. J. Wonham, T. de Camino-Beck, M. A. Lewis. An epidemiological model for West Nile virus: invasion analysis and control applications. Proceedings of the Royal Society of London. Series B: Biological Sciences, 271(1538):501-507, 2004. 